\title{
miR-335 promotes stress granule formation to inhibit apoptosis by targeting ROCK2 in acute ischemic stroke
}

\author{
WENWEN SI*, SHANYU YE*, ZHENXING REN, XIN LIU, ZIMEI WU, YI LI, JIANHONG ZHOU, \\ SAIXIA ZHANG, YIWEI LI, RUDONG DENG and DONGFENG CHEN \\ Department of Anatomy, The Research Center of Basic Integrative Medicine, \\ Guangzhou University of Chinese Medicine, Guangzhou, Guangdong 510006, P.R. China
}

Received August 11, 2018; Accepted January 16, 2019

DOI: 10.3892/ijmm.2019.4073

\begin{abstract}
Under harmful environmental conditions, stress granules (SGs), macromolecular aggregates that are associated with cell survival and death, are produced in the eukaryotic cytoplasm. However, whether and how microRNAs (miRNAs/miRs) modulate SG formation induced by acute ischemic stroke has not been investigated. In the present study, a rat model of middle cerebral artery occlusion (MCAO) was utilized and miRNA array profiling and reverse transcription-quantitative polymerase chain reaction were performed. The results revealed that miR-335 was downregulated during acute ischemic stroke, which was concomitant with reduced SG formation, enhanced apoptosis levels and increased Rho associated protein kinase 2 (ROCK2) expression. In the MCAO rat and serum-free cell models, miR-335 treatment upregulated SG formation, alleviated the ischemia-induced infarction, and decreased ROCK2 protein expression and apoptosis levels. By contrast, when compared with miR-335 treatment, the inhibition of miR-335 resulted in reduced SG formation and higher ROCK 2 expression and apoptosis levels. Target prediction analysis and luciferase 3 '-untranslated region reporter assay identified ROCK2 as the direct target of miR-335. Furthermore, ROCK2 silencing enhanced SG formation and attenuated the level of apoptosis in the serum-free cell model. In addition, ROCK2 silencing markedly inhibited the effect of miR-335 on SG formation and apoptosis levels. Unexpectedly, the phosphorylation of T-cell intracellular antigen-1 was significantly inhibited by miR-335 in the MCAO rat model, which provides a reasonable
\end{abstract}

Correspondence to: Professor Dongfeng Chen, Department of Anatomy, The Research Center of Basic Integrative Medicine, Guangzhou University of Chinese Medicine, 232 Waihuan East Road, Guangzhou, Guangdong 510006, P.R. China

E-mail: cdf27212@21cn.com

*Contributed equally

Key words: stress granules, miR-335, apoptosis, Rho-associated protein kinase 2 , acute ischemic stroke explanation for the promotional effect of miR-335 on SG formation by specifically targeting ROCK 2 . In conclusion, these results demonstrate that miR-335 promotes SG formation and inhibits apoptosis by reducing ROCK2 expression in acute ischemic stroke, which provides a possible therapeutic target for brain injury.

\section{Introduction}

Stress granules (SG) are a type of complex and dynamic foci that are generated in the cytoplasm when eukaryotic cells undergo different types of stress including endoplasmic reticulum stress, heat shock, arsenite and acute energy starvation (1). The core components of SGs include transcription factors, non-translational mRNAs, nucleating factors and RNA binding proteins (RBPs) (2). When eukaryotes undergo different types of stress, the $\alpha$-subunit of eukaryotic translation initiation factor 2 (eIF2 $\alpha$ ) is phosphorylated, which triggers the formation of SGs (3). Following the phosphorylation of eIF2 $\alpha$, multiple RBPs including T-cell intracellular antigen-1 (TIA1), Ras-GAP $\mathrm{SH}_{3}$ domain-binding protein 1 and human antigen R self-aggregate to form the SG (4). SG formation efficiently protects important mRNAs and proteins from degradation and misfolding, which enhances cellular resistance to apoptosis (5).

Acute ischemic stroke (AIS) is the main cause of adult physical disability and mortality worldwide (6), and the process of pathogenesis involves energy failure, inflammation, excitotoxicity, oxidative stress injury and apoptosis (7). An increasing number of reports have demonstrated that apoptosis has a close association with the pathophysiology of AIS (8). Recent studies have indicated that AIS triggers the apoptotic signaling cascade, which leads to a significant level of neuronal death $(9,10)$. Therefore, it's meaningful to investigate the association between SG and apoptosis in order to understand the disease progression of AIS.

Rho associated protein kinase 2 (ROCK2) is a $160 \mathrm{kDa}$ serine/threonine kinase that possesses three different types of functional domains (11). ROCK2 is particularly abundant in brain tissues, which suggests that ROCK2 may perform a key function in cerebral physiological processes (12). According to previous studies, ROCK2 is involved in multiple cellular processes including vascular inflammation and reconstruction, cell adhesion, cell motility and apoptosis (13-15). In addition, ROCK2 has 
been reported to have a connection with a number of diseases, including cardiac hypertrophy, hypertension, atherosclerosis and ischemic stroke (16-18). However, the role of ROCK2 in the process of SG formation remains poorly understood.

MicroRNAs (miRNAs/miRs) are short non-coding RNAs that combine with the $3^{\prime}$ non-translated region of mRNAs and repress the expression of targeted mRNAs (19). Previous reports have demonstrated that miRNAs perform important functions in the pathogenesis of stroke (20-22), including apoptosis, cell cycle progression and cell proliferation (23). miRNAs act as pivotal mediators in the neuronal apoptosis of ischemic stroke by modulating the translation of apoptosis-associated proteins (24-27). Therefore, investigating the apoptotic mechanism of miRNAs in AIS is of great significance. In the present study, rat middle cerebral artery occlusion (MCAO) and serum-free cell models were used to investigate the anti-apoptosis effect of miRNAs and the correlation between SG formation and apoptosis in AIS.

\section{Materials and methods}

Animals and materials. A total of 108 adult male Sprague-Dawley (SD; 250-280 g; 7-8 weeks) rats were purchased from the Animal Center of Guangzhou University of Chinese Medicine (Guangzhou, China). The experiments were approved by the Care and Use of Experimental Animals Committee of Guangzhou University of Chinese Medicine and performed according to the National Institute of Health Guide for the Care and Use of Laboratory Animals. All animals were allowed to acclimate for 1 week prior to MCAO surgery. Upon receipt and throughout the study, the animals were conditioned to the same environment during the study. Food and water were available ad libitum. Room humidity (50-70\%) and temperature $\left(21-25^{\circ} \mathrm{C}\right)$ were maintained consistently. In addition, the study room was maintained on a 12-h light/dark cycle.

MCAO model preparation and intracerebroventricular injection (ICV) of mimic and inhibitor. Under anesthesia $(100 \mathrm{mg} / \mathrm{kg}$ ketamine and $10 \mathrm{mg} / \mathrm{kg}$ xylazine, intraperitoneal injection), rats were subjected to $2 \mathrm{~h}$ MCAO surgery using intraluminal suture as described in a previous report (28). Body temperature was kept at $\sim 36.5^{\circ} \mathrm{C}$ with a heating pad during the surgical process until the animal recovered from anesthesia. The animals were allowed to regain full consciousness in a nursery cage, which was heated $\left(\sim 30^{\circ} \mathrm{C}\right)$ and oxygenated prior to returning to their normal cage. After MCAO surgery, rats immediately underwent ICV of miR-335 mimic and inhibitor under anesthesia. The rats were fixed and one small hole was drilled into the skull of the ischemic hemisphere, in order to deliver the miR-335 mimic and inhibitor into the lateral ventricle. The stereotaxic coordinates for ICV injection were as follows: $-0.9 \mathrm{~mm}$ anterior, $1.8 \mathrm{~mm}$ lateral and $-3.8 \mathrm{~mm}$ from the bregma. A total of 48 male SD rats were randomly divided into four groups ( $\mathrm{n}=12$ rats/group): Control group (normal saline), Model group (MCAO + normal saline), Mimic group (MCAO + miR-335 mimic, 100 pmol/rat) and Inhibitor group (MCAO + miR-335 inhibitor, $200 \mathrm{pmol} / \mathrm{rat}$ ). miR-335 mimic (micrON ${ }^{\mathrm{TM}}$ miRNA agomir; 5'-ucaagagcaauaacgaaaaaugu-3') and miR-335 inhibitor (micrOFF ${ }^{\mathrm{TM}}$ miRNA antagomir; 5'-acauuuuucguuauugcucuuga-3') were purchased from Guangzhou RiboBio Co., Ltd. (Guangzhou, China).
2,3,5-Triphenyltetrazolium chloride (TTC) staining and infarct volume calculation. To observe the dynamic alterations in SG formation, levels of apoptosis and infarct volume in the MCAO model, a total of 36 male SD rats were divided into six groups ( $\mathrm{n}=6$ rats/group): Control group, $0 \mathrm{~h}$ post-reperfusion group, $6 \mathrm{~h}$ post-reperfusion group, $12 \mathrm{~h}$ post-reperfusion group, $24 \mathrm{~h}$ post-reperfusion group and $36 \mathrm{~h}$ post-reperfusion group. Rats from each group were sacrificed with $\mathrm{CO}_{2}$ asphyxiation, and the brains were removed and coronally sectioned into six slices $(2 \mathrm{~mm} / \mathrm{slice})$. The brain slices were stained in a $2 \%$ solution of TTC (Sigma-Aldrich; Merck KGaA, Darmstadt, Germany) for $20 \mathrm{~min}$ at $37^{\circ} \mathrm{C}$; the unstained area was defined as the infarct area and the infarct area volume was calculated with ImageJ software (Version 14.8; National Institutes of Health, Bethesda, MD, USA). Considering edema of the infarct hemisphere, the percentage of infarct area was calculated as follows: Infarct area $(\%)=$ [contralateral hemisphere area-(ipsilateral hemisphere area-infarct area)]/contralateral hemisphere area $\mathrm{x} 100 \%$.

Terminal deoxynucleotidyl transferase dUTP nick end labeling (TUNEL) staining. Following $24 \mathrm{~h}$ of reperfusion, rats were sacrificed by $\mathrm{CO}_{2}$ asphyxiation. The cortices were washed with cold normal saline and fixed with $4 \%$ paraformaldehyde solution at $4^{\circ} \mathrm{C}$ overnight. Frozen sections (20 $\mu \mathrm{m}$ thickness) were used in TUNEL and immunohistochemical staining. TUNEL staining was conducted following the manufacturer's protocol (Boster Biological Technology, Ltd., Wuhan, China). The percentage of positive cells in the pathological images captured was calculated with Image Pro Plus software (version 6.0; Media Cybernetics, Inc., Rockville, MD, USA). The histopathological observations were documented using a light microscope (Leica DMI400; Leica Microsystems GmbH, Wetzlar, Germany) at x400 magnification and photographed. Each experiment was replicated 3 times and the data were presented as the mean \pm standard error of the mean (SEM)

Immunohistochemical staining. Following peroxidase blocking for $30 \mathrm{~min}$ and $5 \%$ bovine serum albumin (BSA; Sigma-Aldrich; Merck KGaA) blocking for $2 \mathrm{~h}$ at room temperature, frozen sections $(20 \mu \mathrm{m})$ of the brain were incubated with primary antibody (cat. no. ab140595; rabbit anti-TIA1 antibody 1:1,000; Abcam, Cambridge, MA, USA) overnight. Then these sections were incubated with secondary antibody (cat. no. ab6721; horseradish peroxidase-conjugated goat anti-rabbit; 1:5,000; Abcam) for $2 \mathrm{~h}$ at room temperature. The expression of TIA1 was detected using a Streptavidin Peroxidase Immunohistochemical staining kit (Tiangen Biotech Co., Ltd., Beijing, China) with DAB staining. The ratio of positive cells was calculated using Image Pro Plus software (version 6.0; Media Cybernetics, Inc.). The histopathological observations were documented by light microscopy (Leica DMI400; Leica Microsystems) at 400x magnification and images were captured. Each experiment was replicated 3 times and the data are presented as the mean \pm SEM.

miRNA microarray assay. A total of 24 male SD rats were randomly divided into control and model groups $(n=12$ rats/group) to perform miRNA microarray assay and reverse transcription-quantitative polymerase chain reaction 
(RT-qPCR). The cerebral cortices were removed from the Control and Model group rats for the miRNA microarray assay. Total RNA (600 ng) was extracted from the cortices of the different groups using TRIzol reagent (Invitrogen; Thermo Fisher Scientific, Inc., Waltham, MA, USA). The subsequent steps of the experiment were completed by Guangzhou RiboBio Co., Ltd., according to the manufacturer's protocol. Each experiment was replicated 3 times and the data are presented as the mean \pm SEM.

Extraction of totalRNA and RT-qPCR. TotalRNA was extracted from the cerebral cortex using TRIzol reagent (Invitrogen; Thermo Fisher Scientific, Inc.) according to the manufacturer's protocol. The concentration and purity of RNA were determined using spectrophotometers (NanoDrop ND-2000; Thermo Fisher Scientific, Inc.). The specific stem-loop primer of miR-335 was used in RT-PCR and the U6 gene was used as the reference. The following primers were used: miR-335, RT primer 5'-GTCGTATCCAGTGCAGGGTCCGAGGTA TTCGCACTGATACGACACATTT-3', forward 5'-CGGCGC TCAAGAGCAATAACGAA-3', and reverse 5'-ATCCAG TGCAGGGTCCGAGG-3'; U6, RT primer 5'-GTCGTATCC AGTGCAGGGTCCGAGGTATTCGCACTGGATACGACA AAATA-3', forward 5'-AGAGAAGATTAGCATGGCCCC TG-3', and reverse 5'-ATCCAGTGCAGGGTCCGAGG-3'. The reverse transcription was performed by the PrimeScript RT reagent kit (Takara, Biotechnology, Co., Ltd., Dalian, China) according to the manufacturer's protocol, at $37^{\circ} \mathrm{C}$ for $15 \mathrm{~min}$ and $85^{\circ} \mathrm{C}$ for $30 \mathrm{sec}$. Light Cycler $480 \mathrm{SYBR}$ Green I Master (Roche Diagnostics, GmbH, Mannheim, Germany) was applied for fluorescent qPCR. The amplification process was as follows: Denaturation at $95^{\circ} \mathrm{C}$ for $10 \mathrm{~min}$, followed by 35 cycles of denaturation at $95^{\circ} \mathrm{C}$ for $15 \mathrm{sec}$, annealing at $60^{\circ} \mathrm{C}$ for $30 \mathrm{sec}$ and extension at $72^{\circ} \mathrm{C}$ for $1 \mathrm{~min}$. Detection of PCR products was performed using the CFX96 RT-qPCR instrument (Bio-Rad Laboratories, Inc., Hercules, CA, USA). Each experiment was replicated 3 times and the data were presented as the mean \pm SEM.

Dual luciferase reporter system analysis. Bioinformatics software (TargetScan, www.targetscan.org; miRBase, www. mirbase.org) was used to predict the target gene of miR-335 and a dual luciferase reporter system was used to confirm the targeting. First pLUC-ROCK2-wild-type (WT) and pLUC-ROCK2-mutant (MUT) plasmid (cat. no. U5679-02, U5679-03; Shenzhen Huaan Ping Kang Bio Technology, Inc., Shenzhen, China) was constructed. Using Lipofectamine ${ }^{\circledR}$ 2000 (Invitrogen; Thermo Fisher Scientific, Inc.), PC12 cells [American Type Culture Collection, (ATCC), Manassas, VA, USA] were transfected with pLUC-ROCK2 cloning vectors (200 ng), miR-335 mimics (100 nM) or miR-335 negative control $(\mathrm{NC} ; 100 \mathrm{nM})$ for $6 \mathrm{~h}$ in 24 -well plates $\left(5 \times 10^{4}\right.$ cells per well). After $24 \mathrm{~h}$ transfection, the fluorescent value of each well was quantified with Dual-Luciferase ${ }^{\circledR}$ Reporter assay kit (Promega Corporation, Madison, WI, USA), according to the manufacturer's protocol. In the pLUC-ROCK2 plasmid, the target site of rno (Rattus norvegicus)-miR-335 was inserted into the downstream sequence of Renilla luciferase (hRluc). Firefly luciferase was used as the internal reference and for normalization. PC12 cells transfected with pLUC-ROCK2 vector were used as the positive control. Cells transfected with miR-335 NC and pLUC-ROCK2 were regarded as the NC. Each experiment was replicated 3 times and the data are presented as the mean \pm SEM.

Cell culture and the serum-free cell model. PC12 cells were purchased from ATCC and were cultured as described previously (29). The serum-free cell model was established by culturing cells in serum-free medium (RPMI-1640 medium; Gibco; Thermo Fisher Scientific, Inc.) for 0, 6, 12, 18, 24 or $30 \mathrm{~h}$. With arsenite stress serving as the positive control treatment, cells were incubated with sodium arsenite (SA; Sigma-Aldrich; Merck KGaA) at the indicated concentrations $(0.5 \mathrm{mM})$ for $50 \mathrm{~min}$ as previously described (3). The serum-free cell model was only stimulated with serum-free culture medium and did not include the arsenite stress step. After PC12 cells were stimulated for $0-30 \mathrm{~h}$ to ascertain the appropriate duration of stimulation, the expression of TIA1 was detected by immunofluorescent assay as the hallmark of SG production. Each experiment was replicated 3 times and the data are presented as the mean \pm SEM.

Immunofluorescence staining. PC12 cells were grown on glass coverslips in 24 well-plates at $1 \times 10^{5}$ cells per well. According to the experimental protocol, cells were transfected or co-transfected with miR-335 mimic, miR-335 inhibitor, small interfering (si)-RNA-ROCK2 and pLUC-ROCK2 plasmids, and then stimulated with serum-free medium for $24 \mathrm{~h}$. Following fixation with $4 \%$ paraformaldehyde for $30 \mathrm{~min}$ at room temperature, $\mathrm{PC} 12$ cells were permeabilized with $0.3 \%$ Triton X-100 and blocked with 5\% BSA. Then cells were incubated with diluted primary antibody (cat. no. 12133-2-AP; rabbit anti-TIA1 antibody; 1:1,000; ProteinTech Group, Inc.) at $4{ }^{\circ} \mathrm{C}$ overnight. and incubated with the secondary antibody (cat. no. ab150077; Alexa Fluor488-conjugated goat anti-rabbit antibody; 1:5,000; Abcam) for $1 \mathrm{~h}$ in the dark. The PC12 cells were rinsed three times with PBS and stained with DAPI solution (BIOSS, Beijing, China) for nuclear labeling. The staining results were observed with a confocal laser scanning microscope (LSM 800; Carl Zeiss AG, Oberkochen, Germany). The formation of SGs was evaluated by calculating the percentage of positive cells with Image Pro Plus software (Version 6.0; Media Cybernetics, Inc.). Each experiment was replicated 3 times and the data are presented as the mean \pm SEM.

Flow cytometry analysis. PC12 cells were grown in 6 well-plates $\left(5 \times 10^{5}\right.$ cells per well) and divided into different groups including: Control, model, miR-335 mimic, miR-335 inhibitor, siRNA-ROCK2 and NC groups. Each group was transfected with the respective miRNA or siRNA and then incubated with serum-free medium for $24 \mathrm{~h}$ except the control group. Control group was incubated with RPMI-1640 medium containing 10\% fetal bovine serum (Gibco; Thermo Fisher Scientific, Inc.). An Annexin V-FITC Apoptosis Staining/Detection kit (cat. no. ab14085; Abcam) was used to detect the levels of apoptosis. PC12 cells were digested with $0.25 \%$ Trypsin and resuspended with $500 \mu 1$ binding buffer. Annexin V and propidium iodide $(5 \mu \mathrm{l})$ were then added into the suspension and labeled for $5 \mathrm{~min}$ in the dark. The percentage of apoptotic cells was detected using a flow 
cytometer (AccuriC6; BD Biosciences, Franklin Lakes, NJ, USA). Accuri CFlow software (Version 1.0; BD Biosciences) was used to analyze the data. Each experiment was replicated 3 times and the data are presented as the mean \pm SEM.

Transfection of siRNA-ROCK2 in PC12 cells. Three different siRNA-ROCK2 inserts were purchased from Guangzhou RiboBio Co., Ltd., which specifically bound and degraded the ROCK2 mRNA. Using Lipofectamine ${ }^{\circledR} 2000$, PC12 cells were transfected with siRNA-ROCK2-1, siRNA-ROCK2-2 and siRNA-ROCK2-3 $(100 \mathrm{nM})$ for $6 \mathrm{~h}$ in 6 -well plates $\left(5 \times 10^{5}\right.$ cells per well). After $24 \mathrm{~h}$ transfection, western blotting and PCR were used to select the most efficient segment. The sequences of siRNA-ROCK2 were as follows: siRNA-ROCK2-1, 5'-CAT GGTGCATTGTGATACA-3'; siRNA-ROCK2-2,5'-CAGAAG TGCAAGTCTATTA-3'; and siRNA-ROCK2-3, 5'-CT CAAG CTGTGAATAAGTT-3'.

Western blotting analysis. The cerebral cortices of the ischemic brain and PC12 cells were collected for immunoblotting analysis and lysed with radioimmunoprecipitation assay lysis buffer (Thermo Fisher Scientific, Inc.) for $20 \mathrm{~min}$ on ice. The cell debris were removed by centrifuging at $12,000 \mathrm{x} \mathrm{g}$ for $10 \mathrm{~min}$ at $4^{\circ} \mathrm{C}$ and the supernatant was harvested for further evaluation (stored at $-80^{\circ} \mathrm{C}$ ). The bicinchoninic acid method was used to detect protein concentration. Total protein $(30 \mu \mathrm{g})$ was separated by $10 \%$ SDS-PAGE and transferred to $0.45 \mu \mathrm{m}$ polyvinylidene difluoride membranes (EMD Millipore, Billerica, MA, USA). After blocking with 5\% BSA for $2 \mathrm{~h}$ at room temperature, the membranes were incubated with primary antibodies (ROCK2, cat. no. ab71598, 1:1,000; TIA1, cat. no. ab140595, 1:1,000; active caspase-3, cat. no. ab2302, 1:1,000; Bcl-2; cat. no. ab692, 1:1,000; GAPDH, cat. no. ab8245, 1:5,000; Abcam) at $4^{\circ} \mathrm{C}$ overnight. After rinsing with Tris-buffered saline $-0.01 \%$ Tween-20, the membranes were incubated with the secondary antibodies [horseradish peroxidase (HRP)-conjugated goat anti-rabbit, cat. no. ab6721; HRP-conjugated goat anti-mouse, cat. no. ab6789; 1:5,000; Abcam] for $1 \mathrm{~h}$ at room temperature. Enhanced chemiluminescence (ECL) western blotting substrate (Pierce; Thermo Fisher Scientific, Inc.) was applied to visualize the bands. Image acquisition was performed using the Tanon-6200 gel imaging system (Tanon Science and Technology Co., Ltd., Shanghai, China). Image processing software (ImageJ; Version 14.8; National Institutes of Health) was used to analyze the images. Each experiment was replicated 3 times and the data are presented as the mean \pm SEM.

Immunoprecipitation assays. According to the manufacturer's protocol of the Direct Magnetic IP/Co-IP kit (Pierce; Thermo Fisher Scientific, Inc.), the protein extracted from the rat brain tissues was quantified by a bicinchoninic acid assay following addition of phosphatase and protease inhibitors. A total of $500 \mu \mathrm{g}$ protein $(1-2 \mathrm{mg} / \mathrm{ml})$ was incubated with $25 \mu \mathrm{l}$ TIA1-coupled magnetic beads for $2 \mathrm{~h}$ at room temperature on a rotator. After washing and eluting the binding beads, TIA1 protein was collected and a western blotting assay was used to detect the phosphorylation levels. ECL western blotting substrate (Pierce; Thermo Fisher Scientific, Inc.) was used to visualize the bands. Image acquisition was performed with the Tanon-6200 gel imaging system (Tanon Science and Technology Co., Ltd.). Image processing software (ImageJ; National Institutes of Health) was used to analyze the images. Each experiment was replicated 3 times and the data are presented as the mean \pm SEM.

Statistical analysis. The experimental data are presented as the mean \pm SEM. SPSS 22.0 software (IBM Corp., Armonk, NY, USA) was used for statistical analysis. The figures were all produced using GraphPad Prism (Version 6.0; GraphPad Software, Inc., La Jolla, CA, USA). Student's t-test was used to analyze the difference between two groups. The comparison between multiple groups was analyzed using one-way analysis of variance followed by Tukey's multiple comparisons test as the post hoc test. $\mathrm{P}<0.05$ was considered to indicate a statistically significant difference. Each experiment was replicated 3 times.

\section{Results}

$S G$ formation is reduced and apoptosis levels are elevated in MCAO rats following $24 \mathrm{~h}$ of reperfusion. To evaluate the optimal point of reperfusion in the MCAO model, rats subjected to MCAO ( $\mathrm{n}=36$ rats, 6 rats/group) were sacrificed at 0,6 , 12,24 or $36 \mathrm{~h}$ following reperfusion. The brain tissues were harvested and infarct volume was detected by TTC staining. SG formation and apoptosis levels were detected by immunohistochemical and TUNEL staining in frozen brain sections, respectively. The results revealed that SG formation peaked at $6 \mathrm{~h}$ postreperfusion, then progressively decreased until $24 \mathrm{~h}$ and increased at $36 \mathrm{~h}$ (Fig. 1A). Apoptosis levels and infarct volume exhibited similar patterns during different time points following reperfusion, which peaked significantly at $24 \mathrm{~h}$ post-reperfusion and reduced at $36 \mathrm{~h}$ post-reperfusion $(\mathrm{P}<0.0001$; Fig. $1 \mathrm{~B}$ and $\mathrm{C})$. At $24 \mathrm{~h}$ post-reperfusion, SG formation was at the lowest level, whereas apoptosis and infarct volume were at the highest levels. However, at $36 \mathrm{~h}$ post-reperfusion, SG formation was increased, whereas apoptosis and infarct volume were decreased; this may be due to the recovery ability of the rats. These data suggested that MCAO injury may reduce SG formation and elevate apoptosis levels at $24 \mathrm{~h}$. In addition, $24 \mathrm{~h}$ post-reperfusion was the optimal time point to investigate the association between SG formation and apoptosis.

The expression of miR-335 is significantly decreased in $M C A O$ rats. A total of 24 male SD rats were randomly divided into control and model groups (12 rats/group). The MCAO model was established using the suture method. Following $24 \mathrm{~h}$ of reperfusion, all of the rats were sacrificed by $\mathrm{CO}_{2}$ asphyxiation, the detailed procedure were performed as described previously (30). The relative expression of miR-335 was measured by RT-qPCR (Fig. S1A). Neurological deficit scoring was performed to evaluate the model. The whole brains were removed for TTC staining. Compared with the control group, the infarct volume and behavioral score significantly increased in the model group $(\mathrm{P}<0.001$ and $\mathrm{P}=0.0026$; Fig. $2 \mathrm{~A}$ and $\mathrm{B})$, which verified the success of MCAO model establishment. Representative TTC-stained coronal sections of each group were presented in Fig. 2C. An miRNA microarray assay was performed to determine the miRNAs with specific expression 
A

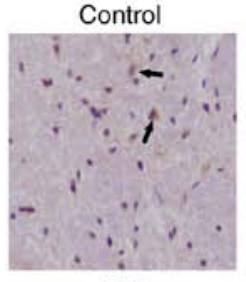

$12 \mathrm{~h}$

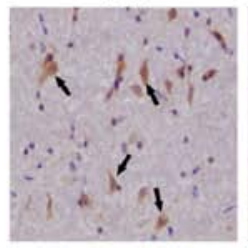

B

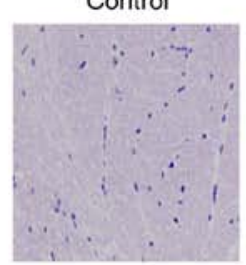

$12 \mathrm{~h}$

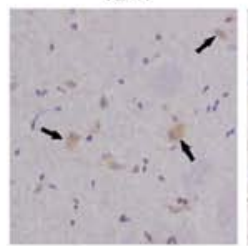

C

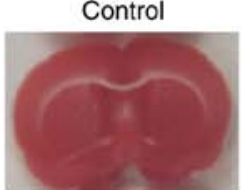

$12 \mathrm{~h}$

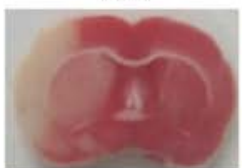

$\mathrm{Oh}$

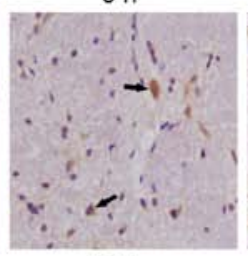

$24 \mathrm{~h}$

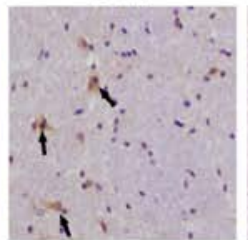

$\mathrm{Oh}$

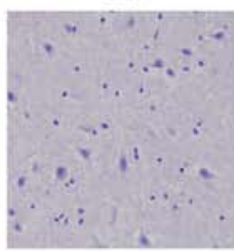

$24 \mathrm{~h}$

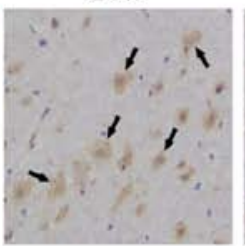

$\mathrm{Oh}$

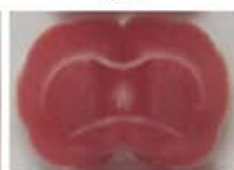

$24 \mathrm{~h}$

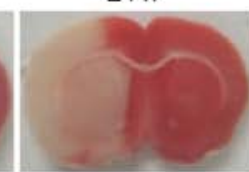

$6 \mathrm{~h}$

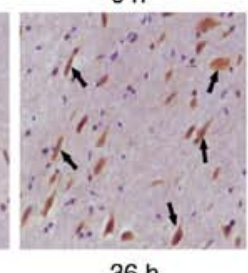

$36 \mathrm{~h}$

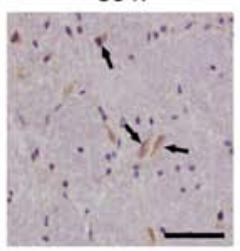

$6 \mathrm{~h}$

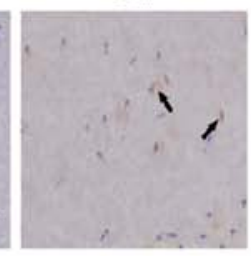

$36 \mathrm{~h}$

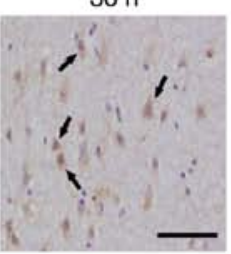

$6 \mathrm{~h}$

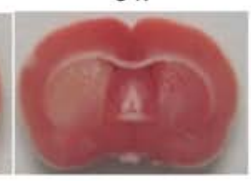

$36 \mathrm{~h}$
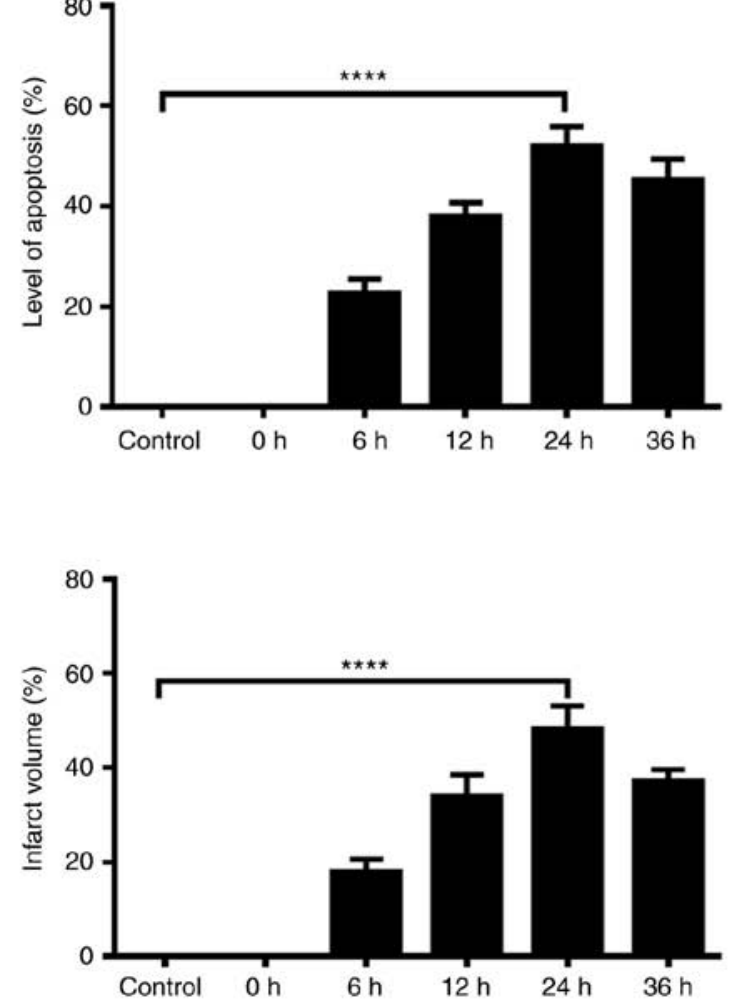

Figure 1. An inverse association between SG formation and apoptosis in a MCAO model following $24 \mathrm{~h}$ reperfusion. (A) SG formation was inhibited in the MCAO model after $24 \mathrm{~h}$ reperfusion. (B) Apoptosis levels were elevated in MCAO rats following $24 \mathrm{~h}$ reperfusion. (C) Infarct volume was the greatest following $24 \mathrm{~h}$ reperfusion in the MCAO model. TTC staining was used for the measurement of infarct size. Data are presented as the mean \pm standard error of the mean. ${ }^{* * *} \mathrm{P}<0.001$ and ${ }^{* * * *} \mathrm{P}<0.0001$ vs. the control group; ${ }^{\#} \mathrm{P}<0.056 \mathrm{~h}$ vs. $24 \mathrm{~h}$ (one-way analysis of variance test). Each experiment was replicated 3 times. The control group underwent sham surgery (the common carotid artery of rats in control group was isolated with no ligation). Scale bars, $25 \mu \mathrm{m}$. SG, stress granules; MCAO, middle cerebral artery occlusion.

in the ischemic cortices of rats. When compared with the control group, there were 20 miRNAs which included 4 upregulated miRNAs and 16 downregulated miRNAs that exhibited differential expression in the model group. miR-335 exhibited the most obvious differential expression in downregulated miRNAs (Fig. 2D). This experimental result was confirmed by the qPCR assay of multiple miRNAs (miR-335, miR-361-3p, miR-290, miR-292-3p, miR-296-5p and miR-3473) in the two groups. Compared with the control group, the miR-335 expression of the model group was significantly decreased $(\mathrm{P}<0.0001$; Fig. 2E). These findings suggested that miR-335 expression was reduced in MCAO rats.
miR-335 treatment increases $S G$ formation and reduces the ischemia-induced injury, ROCK2 protein expression and apoptosis levels in MCAO rats. To investigate the protective effect of miR-335 in vivo, 48 male SD rats were randomly divided into 4 groups (control, model, miR-335 mimic and miR-335 inhibitor groups; 12 rats/group). The MCAO model was established using the suture method in rats. miR-335 mimics or the miR-335 inhibitor were immediately injected into the left lateral ventricle of the rats in the corresponding groups following neurological deficit scoring at $0 \mathrm{~h}$. Following $24 \mathrm{~h}$ of reperfusion, all of the rats were sacrificed and brain tissues were harvested for the subsequent analyses. The TTC 

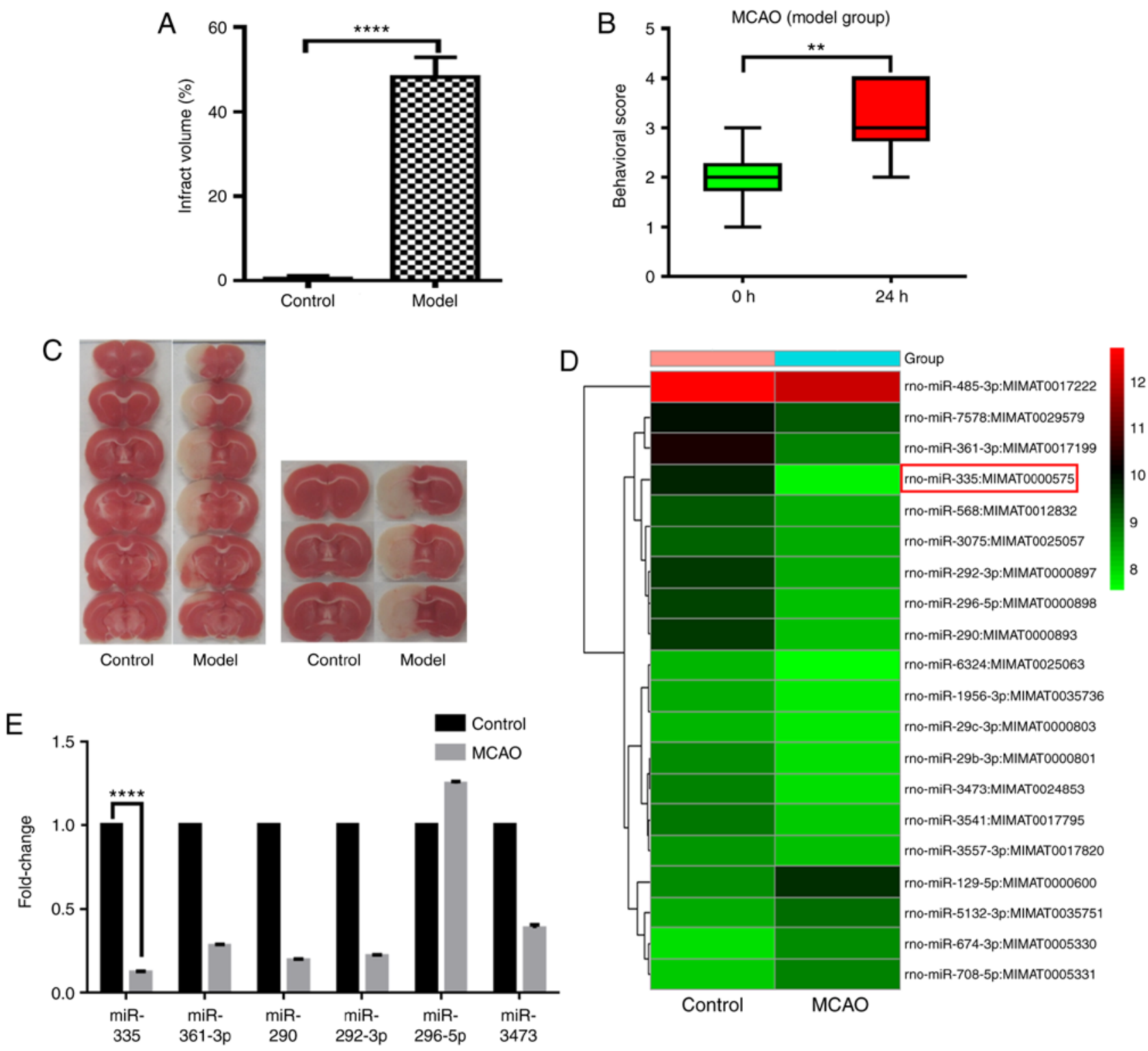

Figure 2. miR-335 expression is significantly decreased in the MCAO model. (A) The infarct volume of the model group was significantly increased compared with the control group. Brain tissues were sectioned into 6 slices of $2-\mathrm{mm}$ thickness. TTC staining was used for the measurement of infarct size. ${ }^{* * * *} \mathrm{P}<0.0001$ vs. the control group (Student's t-test). (B) The neurological score of the MCAO model group was increased significantly at $24 \mathrm{~h}$. The 0 (green) and $24 \mathrm{~h}$ (red) neurological scores of the control group were considered as the zero points. ${ }^{* *} \mathrm{P}<0.01,0 \mathrm{~h}$ vs. $24 \mathrm{~h}$ (Student's t-test). (C) Representative TTC-stained serial sections (left panel) and the most severely damaged section (right panel) of each group. The third slices of the brain were considered to be the most severely damaged sections. (D) The expression of miR-335 was decreased in the miRNA microarray assay. miR-335 was marked with a red rectangle. (E) The expression of rno-miR-335 was significantly reduced in the MCAO model. The qPCR assay of the 6 selected miRNAs (miR-335, miR-361-3p, miR-290, miR-292-3p, miR-296-5p and miR-3473) was performed to determine the differential expression in the MCAO model. Data are presented as the mean \pm standard error of the mean. ${ }^{* * * * *} \mathrm{P}<0.0001$ vs. control group (Student's t-test). Control group was under sham surgery. Each experiment was replicated 3 times. MCAO, middle cerebral artery occlusion; $\mathrm{miR} / \mathrm{miRNA}$, microRNA; rno, Rattus norvegicus.

staining (Fig. 3A) and neurological deficit scores were used to evaluate the MCAO model. Compared with the model group, the infarct volume and behavioral score significantly decreased in the mimic group $(\mathrm{P}=0.0029$ and $\mathrm{P}=0.0104$, respectively; Fig. 3B and C). In addition, when compared with the model group, ROCK2 and caspase-3 expression markedly reduced in the miR-335 mimic group $(\mathrm{P}=0.0107 ; \mathrm{P}=0.0272)$, whereas TIA1 and Bcl-2 expression increased $(\mathrm{P}<0.0001 ; \mathrm{P}=0.0020)$. The immunohistochemical staining of TIA1 was utilized to detect the production of SG. According to the results of immunohistochemistry, the miR-335 mimic significantly promoted TIA1 expression when compared with the model group $(\mathrm{P}<0.0001 ; \mathrm{Fig} .3 \mathrm{E})$. The apoptosis levels were detected by TUNEL staining in frozen brain sections. In contrast to the model group, the miR-335 mimic significantly reduced the apoptotic levels of the ischemic brain ( $\mathrm{P}=0.0236$; Fig. 3F). These results indicated that miR-335 increased SG formation and reduced the ischemia-induced injury, ROCK2 protein expression and apoptosis levels in MCAO rats. By analyzing the data, the present study revealed that there was a negative correlation between ROCK2 and TIA1 expression in vivo.

miR-335 inhibits the phosphorylation level of TIA1 in a MCAO rat model. To study the potential mechanism of miR-335 


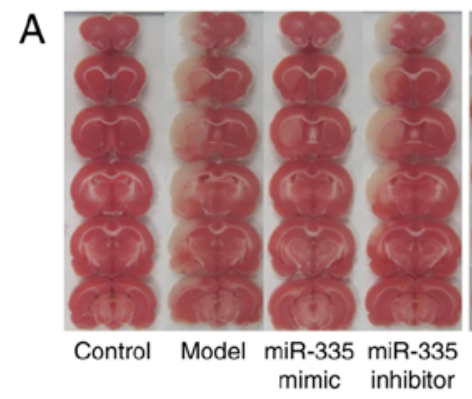

C

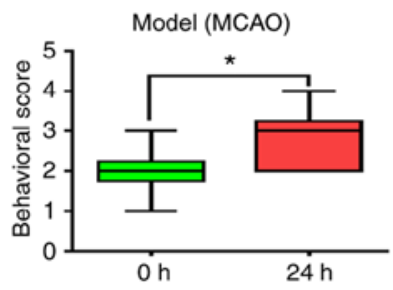

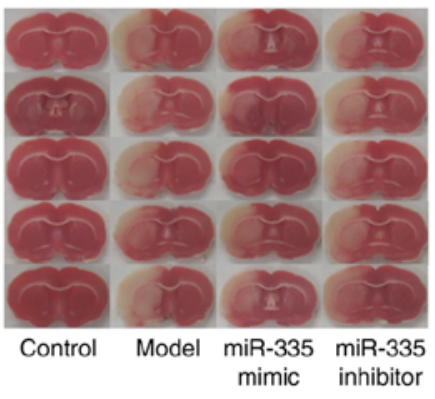

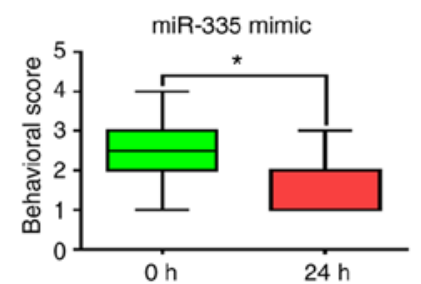

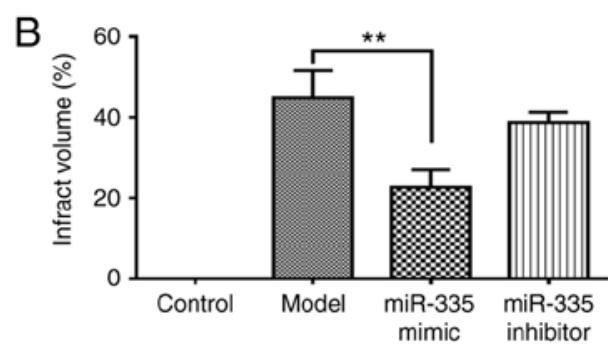

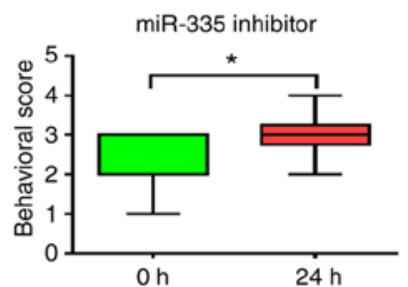

D
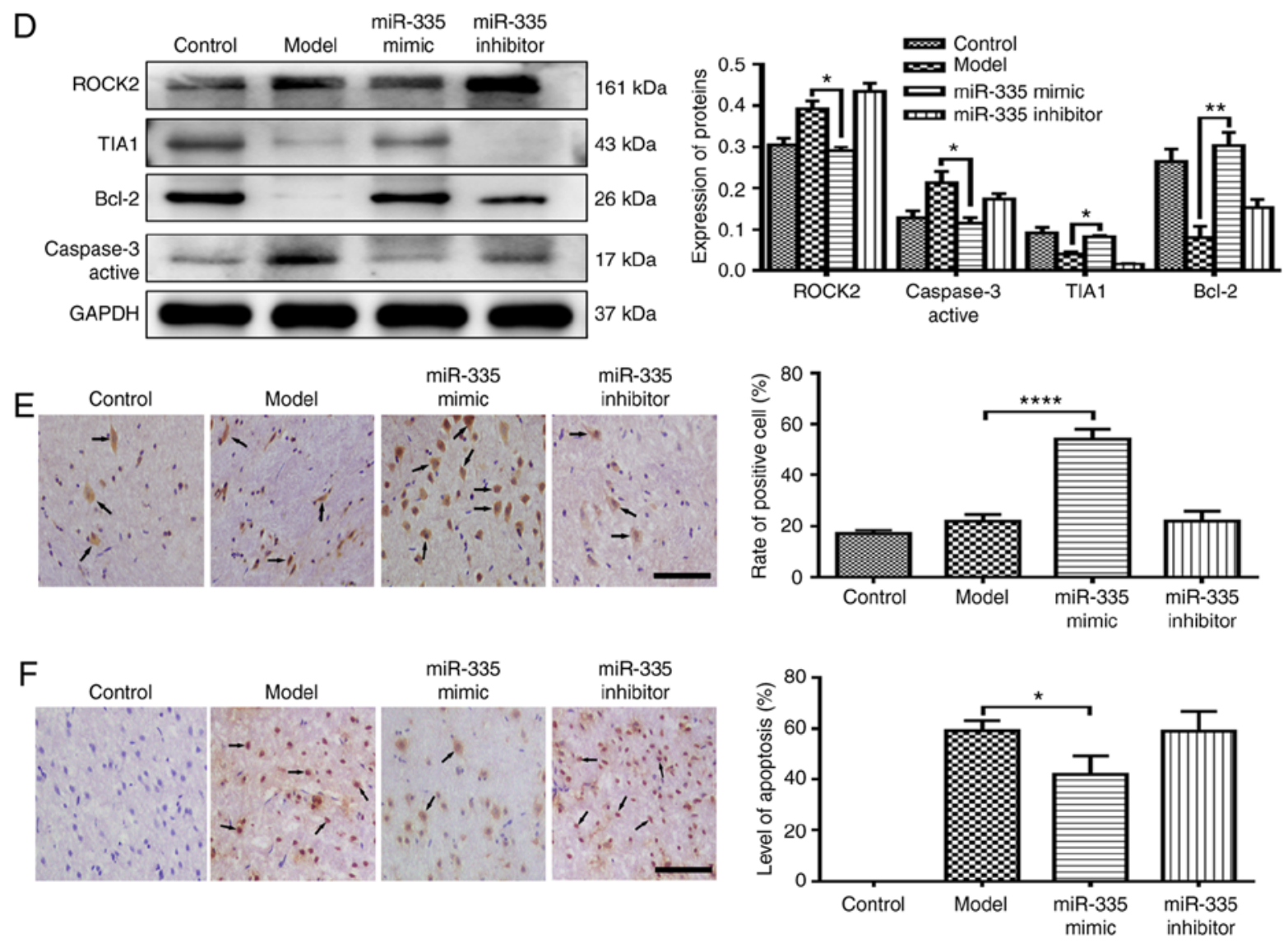

Figure 3. miR-335 treatment increases SG formation and reduces ischemia-induced brain damage, ROCK2 protein expression and apoptosis levels. (A) Representative TTC-stained coronal sections of each group. Serial sections are shown in the left panel, the third sections are shown in right panel. (B) miR-335 reduced infarct volume in the MCAO model. TTC staining was used for the measurement of infarct size. ${ }^{* *} \mathrm{P}<0.01$, vs. model group (one-way ANOVA test). (C) miR-335 reduced neurological deficits scores in MCAO model. " $\mathrm{P}<0.05,0 \mathrm{~h}$ vs. $24 \mathrm{~h}$ (Student's t-test). (D) miR-335 reduced ROCK2 and caspase 3 expression and increased TIA1 and Bcl-2 expression in MCAO rats (6 rats/group). " $\mathrm{P}<0.05$ and ${ }^{* *} \mathrm{P}<0.01$ vs. model group (one-way ANOVA test). (E) miR-335 promoted SG formation in MCAO rats (6 rats/group). ${ }^{* * * * *} \mathrm{P}<0.0001$ vs. model group (one-way ANOVA test). (F) miR-335 suppressed the apoptosis levels in the MCAO model. TUNEL staining in frozen sections was used to evaluate the levels of apoptosis. Data were presented as the mean \pm standard error of the mean. "P $<0.05$ vs. model group (one-way ANOVA test). Each experiment was replicated 3 times. Scale bars, $25 \mu \mathrm{m}$. Control group underwent sham surgery. SG, stress granules; MCAO, middle cerebral artery occlusion; miR, microRNA; ROCK2, Rho associated protein kinase 2; TIA1, T-cell intracellular antigen-1; Bcl-2, B-cell lymphoma 2; ANOVA, analysis of variance.

promoting SG assembly, the present study conducted immunoprecipitation to detect the phosphorylation level of TIA1 in the ischemic cortex of MCAO rats. As presented in Fig. 4A, TIA1 phosphorylation was increased in the model and inhibitor groups when compared with control group. In addition, TIA1 phosphorylation was significantly inhibited in the miR-335 mimic group compared with the model $(\mathrm{P}=0.0011)$. The mechanism underlying how miR-335 promotes SG formation 
A

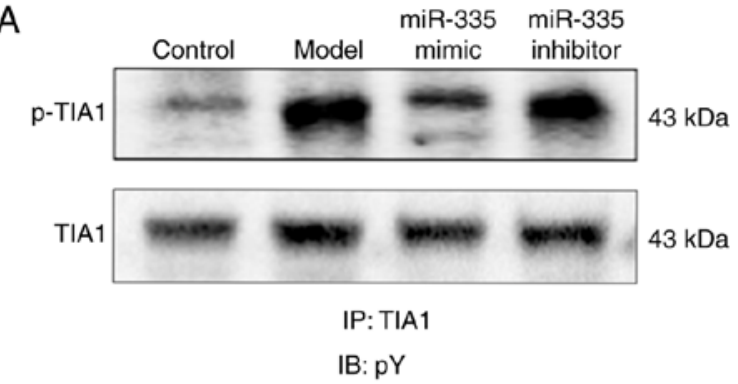

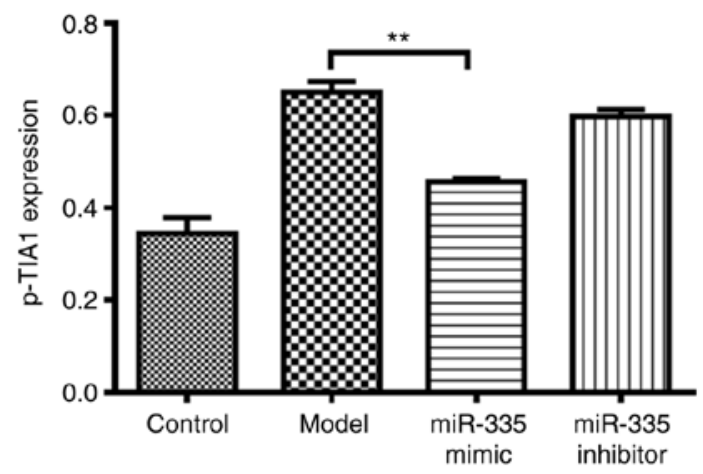

B

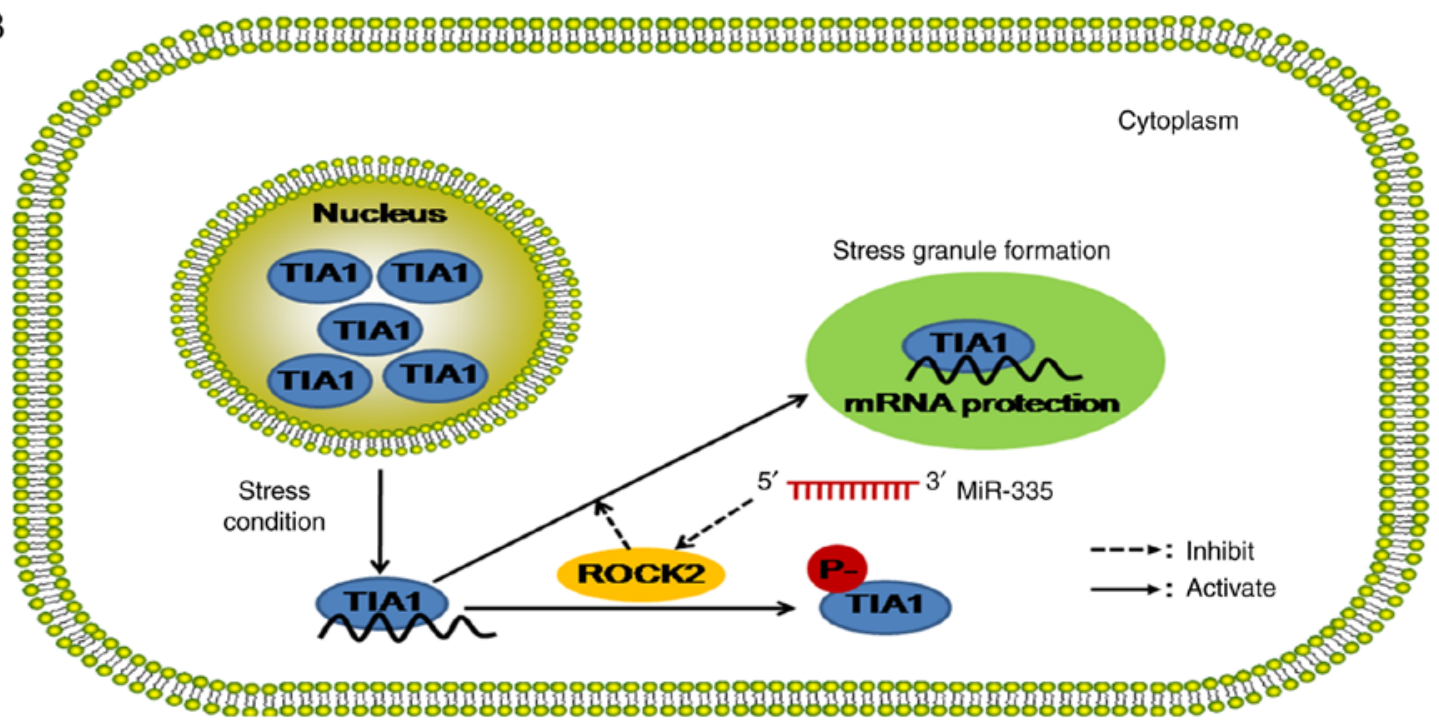

Figure 4. miR-335 inhibits the phosphorylation level of TIA1 in the MCAO rat model. (A) TIA1 phosphorylation was significantly decreased by miR-335 in the immunoprecipitation assay. Protein extracted from the rat brain tissue was incubated with TIA1 coated beads and a western blotting assay was used to detect the levels of phosphorylation. Data were presented as the mean \pm standard error of the mean ( 6 rats/group). ${ }^{* *} \mathrm{P}<0.01$ vs. the model group (one-way analysis of variance test). (B) Schematic representation of the proposed effect of miR-335 on ROCK2 activity and TIA1 phosphorylation in SG formation. Control group underwent sham surgery. TIA1, T-cell intracellular antigen-1; MCAO, middle cerebral artery occlusion; miR, microRNA; ROCK2, Rho associated protein kinase 2.

and the regulatory relationship between ROCK2 and TIA1 phosphorylation is presented in Fig. 4B. These results indicated that miR-335 inhibits the phosphorylation level of TIA1 by decreasing the kinase activity of ROCK 2 in MCAO rats.

miR-335 directly targets the 3'UTR of ROCK2 mRNA in PC12 cells. Using bioinformatics software (TargetScan, www. targetscan.org; miRBase, www.mirbase.org), the present study demonstrated that there were predicted binding sites of miR-335 in the 3'-UTR of ROCK2 mRNA (Fig. 5A). To verify this result, a dual luciferase reporter system assay was performed in PC12 cells. Cells transfected with miR-335 NC and pLUC-ROCK2 WT plasmid were treated as the NC. The plasmid profile is presented in Fig. 5B. Compared with the NC group, the relative luciferase activity of cells co-transfected with pLUC-ROCK2 plasmid and miR-335 mimic was significantly decreased $(\mathrm{P}=0.0022$; Fig. 5C). These results confirm that the 3'-UTR of ROCK 2 mRNA may be the target of miR-335 in PC12 cells.

miR-335 promotes $S G$ formation, reduces ROCK2 expression and suppresses cell apoptosis in serum-free cells. To investigate the protective effect of miR-335 in vitro, a serum-free cell model was used in the present study. The stimulatory time experiments indicated that $24 \mathrm{~h}$ stimulation generated the most obvious SG formation (Fig. 6A). Then the PC12 cells were cultured in 6/24-well plates and divided into 6 groups: Control, model, miR-335 mimic, miR-335 NC, miR-335 inhibitor and miR-335 inhibitor NC groups. The relative expression of miR-335 was measured by RT-qPCR (Fig. S1B). Following transfection with an miR-335 mimic, mimic NC, inhibitor and inhibitor NC respectively, the cells were cultured with serum-free stimulation for $24 \mathrm{~h}$ in RPMI-1640 medium. As presented in Fig. 6B, compared with the model group, ROCK2 expression significantly decreased and TIA1 expression increased significantly in the miR-335 mimic group $(\mathrm{P}<0.0001 ; \mathrm{P}<0.0001)$. According to the results of immunofluorescence staining (Fig. 6C), the miR-335 mimic significantly promoted SG production $(\mathrm{P}<0.0001)$. As presented in Fig. 6D, compared with the model group, apoptosis levels were significantly reduced in the miR-335 mimic group $(\mathrm{P}<0.0001)$. These results indicated that miR-335 promoted SG formation, decreased ROCK2 expression and prevented cell apoptosis in the serum-free cell model.

ROCK2 silencing enhances $S G$ formation and reduces the apoptosis level in serum-free cells. To analyze the effect of ROCK2 in vitro, the present study performed RNA interference (RNAi) to silence the expression of ROCK2 


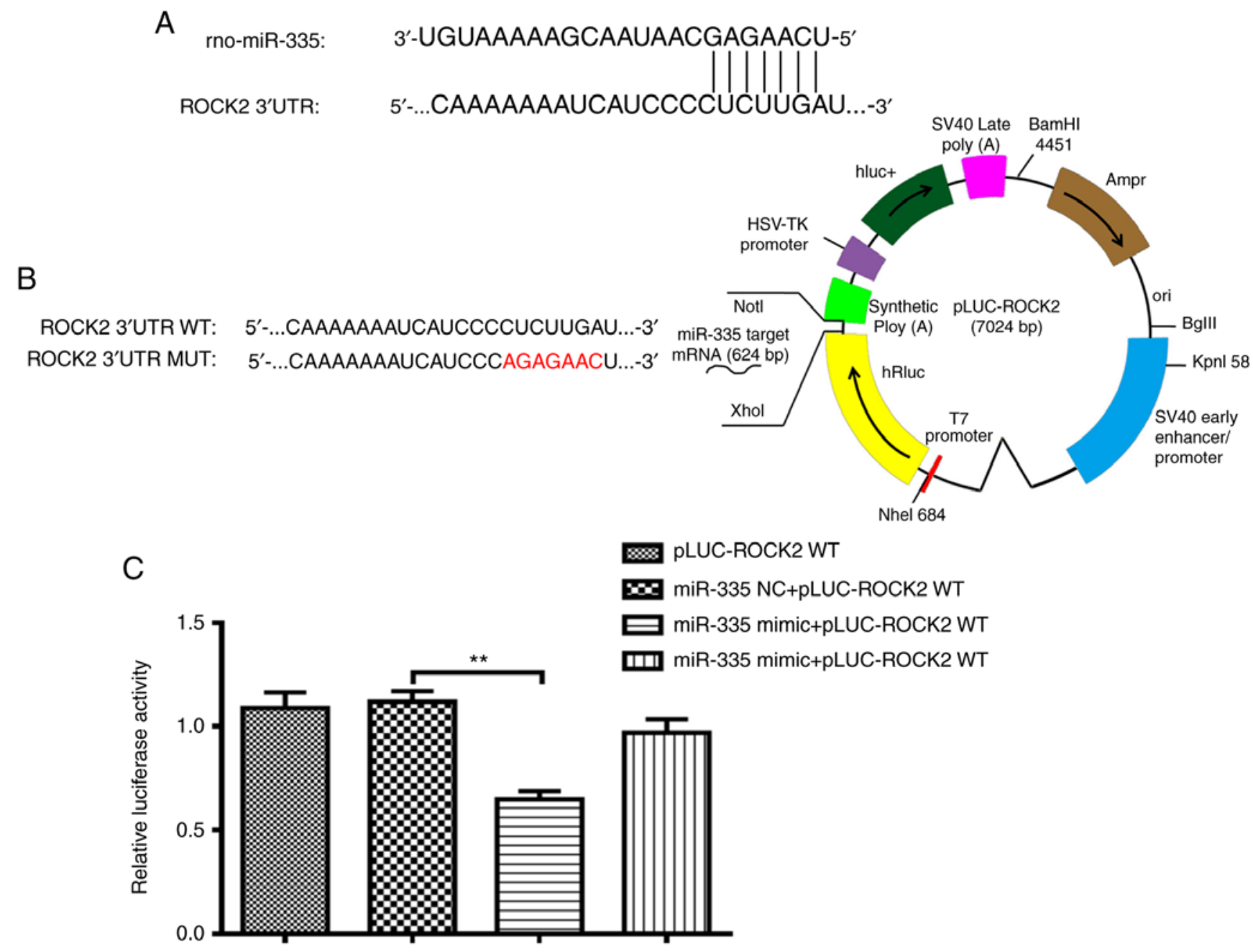

Figure 5. 3'-UTR of ROCK2 was the specific target of miR-335. (A) miR-335 had binding sites in the 3'-UTR of ROCK2. TargetScan and MiRBase were used to search for the target sites. (B) The construction profile of the pLUC-ROCK2 plasmid. The binding sequence in the 3'-UTR of ROCK2 was replaced in the pLUC-ROCK2 MUT plasmid. (C) miR-335 reduced the relative luciferase activity of pLUC-ROCK2. PC12 cells were transfected with pLUC-ROCK2 vector (200 ng), miR-335 mimics (100 nM) or miR-335 NC (100 nM) for $48 \mathrm{~h}$ using Lipofectamine ${ }^{\circledR} 2000 .{ }^{* *} \mathrm{P}<0.05$ vs. miR-335 NC+pLUC-ROCK2 group (One-way analysis of variance test). Data were presented as the mean \pm standard error of the mean. Each experiment was replicated 3 times. Untransfected cells were used as the control group. UTR, untranslated region; ROCK2, Rho associated protein kinase 2; miR, microRNA; NC, negative control; WT, wild type; MUT, mutant.

in serum-free cells. PC12 cells were transfected with siRNA-ROCK2-1, siRNA-ROCK2-2 and siRNA-ROCK2-3 $(100 \mathrm{nM})$ for 6 and $12 \mathrm{~h}$ in 6 -well plates $\left(1 \times 10^{5}\right.$ cells per well) to select the most effective segment and transfection time point. The relative expression of ROCK mRNA was measured by RT-qPCR (Fig. S1C). Western blotting results demonstrated that siRNA-ROCK2-1 transfected for $6 \mathrm{~h}$ was the most effective method to silence ROCK 2 expression (Fig. 7A). Furthermore, compared with the model group, ROCK 2 expression significantly decreased and TIA1 expression significantly increased in the siRNA-ROCK2 group $(\mathrm{P}=0.0013$; $\mathrm{P}=0.0046$; Fig. 7B). As presented in Fig. 7C, in contrast to the model group, $\mathrm{SG}$ formation was increased in the siRNA-ROCK 2 group $(\mathrm{P}=0.0002)$. Flow cytometry analysis was used to evaluate apoptosis levels in the cell model. Compared with the model group, the apoptosis level significantly reduced in the siRNA-ROCK2 group $(\mathrm{P}<0.0001$; Fig. 7D). These results indicated that ROCK2 silencing promoted SG formation, elevated TIA1 protein expression and prevented cell apoptosis in serum-free cells.
ROCK2 silencing significantly attenuates the effect of miR-335 on SG formation and apoptosis. According to the aforementioned results, the present study revealed that miR-335 promoted SG formation and suppressed cell apoptosis in PC12 cells with serum-free stimulation. Previous studies have demonstrated that the target gene can be regulated by multiple miRNAs $(31,32)$. Therefore, to investigate the association between the protective function of miR-335 and ROCK2, co-transfection of miR-335 and siRNA-ROCK2 was performed in the serum-free cell model. As presented in Fig. 8A, compared with the model group, ROCK 2 expression was significantly inhibited in the co-transfection and siRNA-ROCK2 groups $(\mathrm{P}=0.0024$; $\mathrm{P}=0.0035)$, and TIA1 expression was significantly increased in these two groups $(\mathrm{P}=0.0009 ; \mathrm{P}=0.0030)$. Furthermore, there was no significant difference in ROCK2 and TIA1 expression between the siRNA-ROCK2 and co-transfection groups $(\mathrm{P}=0.9885 ; \mathrm{P}=0.7090)$. In contrast to the model group, SG formation was markedly increased in the co-transfection and siRNA-ROCK 2 groups $(\mathrm{P}=0.0019 ; \mathrm{P}=0.0034)$, however, 
A
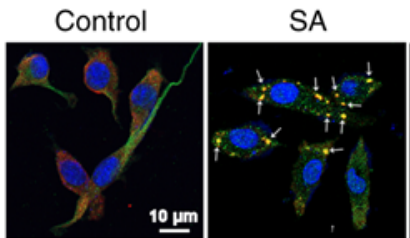

$18 \mathrm{~h}$

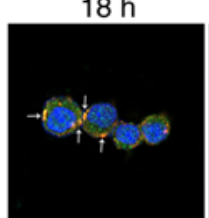

$24 \mathrm{~h}$

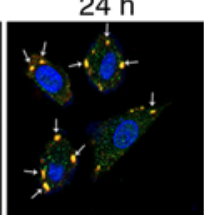

$6 \mathrm{~h}$

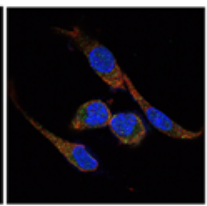

$30 \mathrm{~h}$

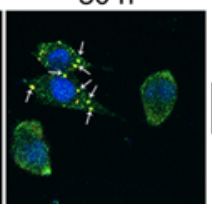

TIA1/ G3BP1/
$12 \mathrm{~h}$

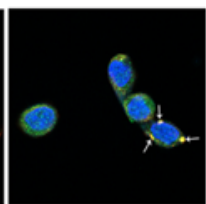

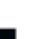

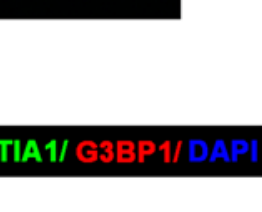

B
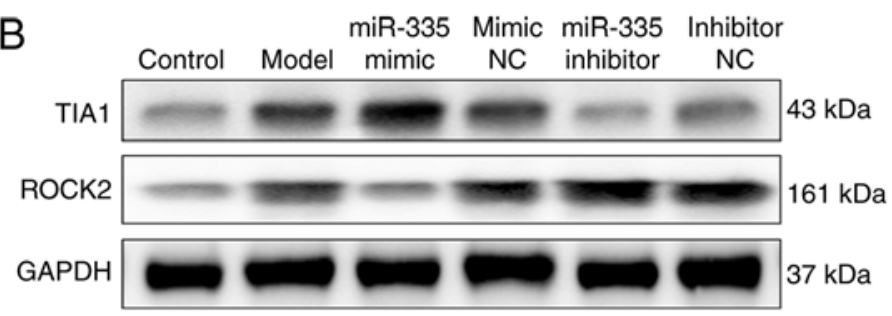

C
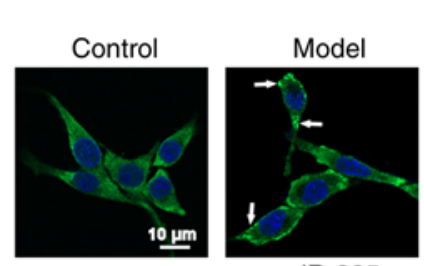

miR-335

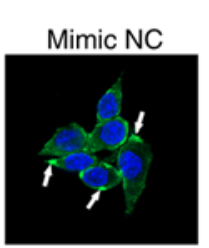

miR-335

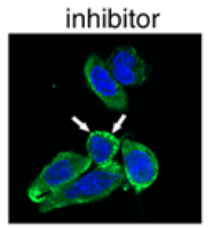

D A01 Control
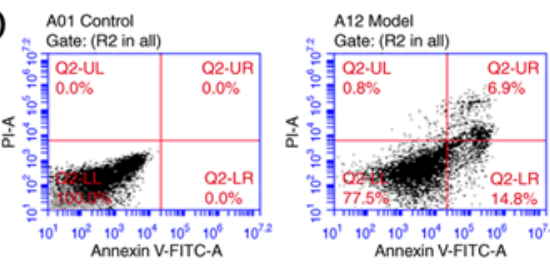

10 Mimic NC
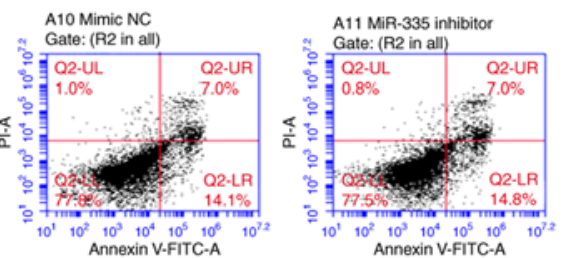

\section{TIA1/}
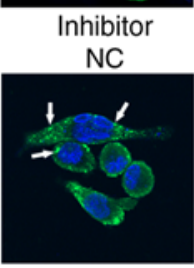
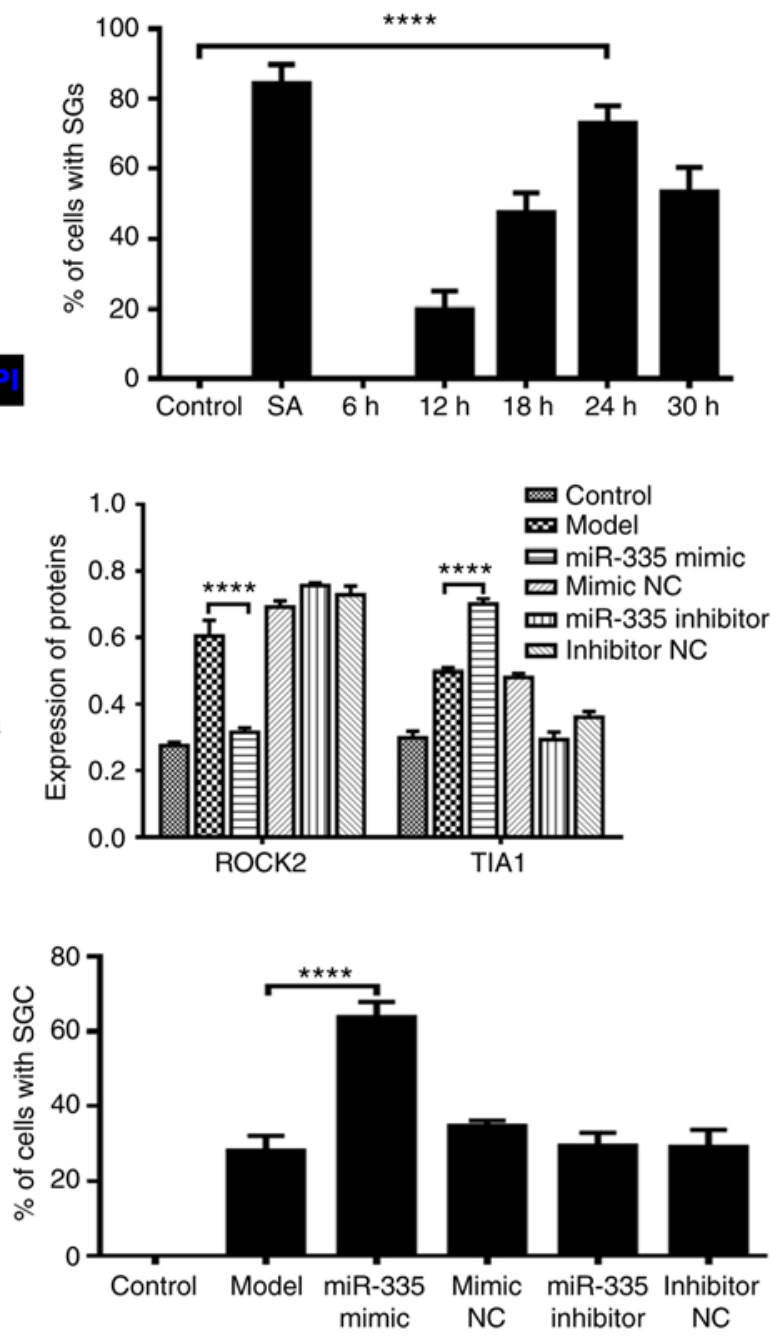

Figure 6. miR-335 promotes SG formation, reduces ROCK2 expression and cell apoptosis in serum-free cells. (A) Stimulation for $24 \mathrm{~h}$ produced the most obvious SG formation in PC12 cells. Immunofluorescence staining was used to observe SG formation. ${ }^{* * * *} \mathrm{P}<0.0001$ vs. the control group. (B) miR-335 reduced ROCK2 expression and increased TIA1 expression in the serum-free cell model. miR-335 mimic and mimic NC was transfected into cells at a $50 \mathrm{nM}$ final concentration and miR-335 inhibitor and inhibitor NC was transfected at a final concentration of $100 \mathrm{nM}$ in 6-wells plate. Untransfected cells were used as the control group. ${ }^{* * * *} \mathrm{P}<0.0001$ vs. the model group. (C) miR-335 promoted SG formation in the serum-free cell model. ${ }^{* * * * *} \mathrm{P}<0.0001$ vs. the model group. (D) miR-335 suppressed cell apoptosis in the serum-free cell model. The apoptotic rate was detected by flow cytometry analysis using an Annexin V-FITC apoptosis detection kit. ${ }^{* * * *} \mathrm{P}<0.0001$ vs. the model group. Data are presented as the mean \pm standard error of the mean. One-way analysis of variance test. Each experiment was replicated 3 times. Scale bars, $10 \mu \mathrm{m}$. miR, microRNA; SG, stress granules; MCAO, middle cerebral artery occlusion; TIA1, T-cell intracellular antigen-1; ROCK2, Rho associated protein kinase 2; NC, negative control; FITC, fluorescein isothiocyanate.

no significant difference was identified between these two groups $(\mathrm{P}=0.9591$; Fig. $8 \mathrm{~B})$. Flow cytometry analysis was used to detect the level of apoptosis. Compared with the model group, the apoptotic level was significantly downregulated in the co-transfection and siRNA-ROCK2 groups $(\mathrm{P}=0.0003 ; \mathrm{P}=0.0008)$, however, no significant difference 
A
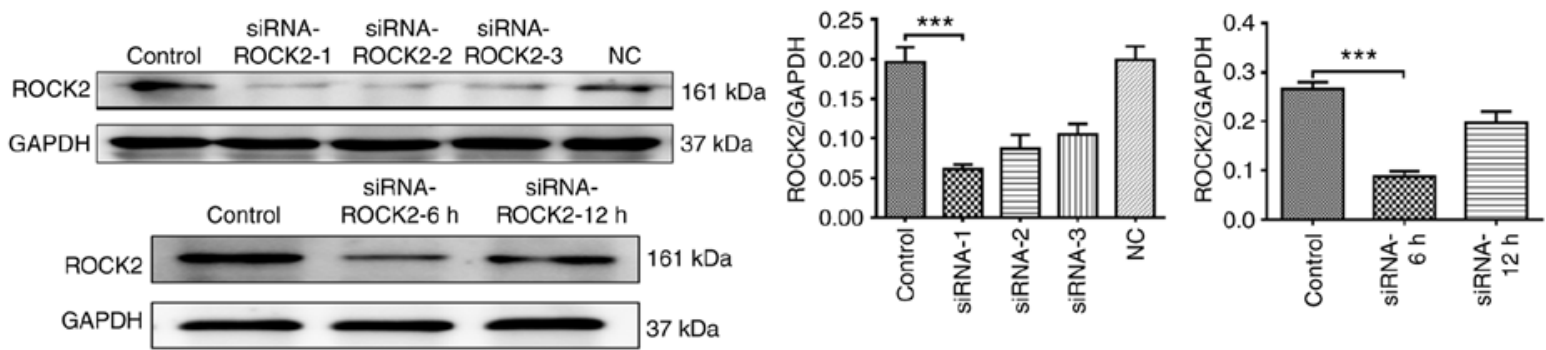

B
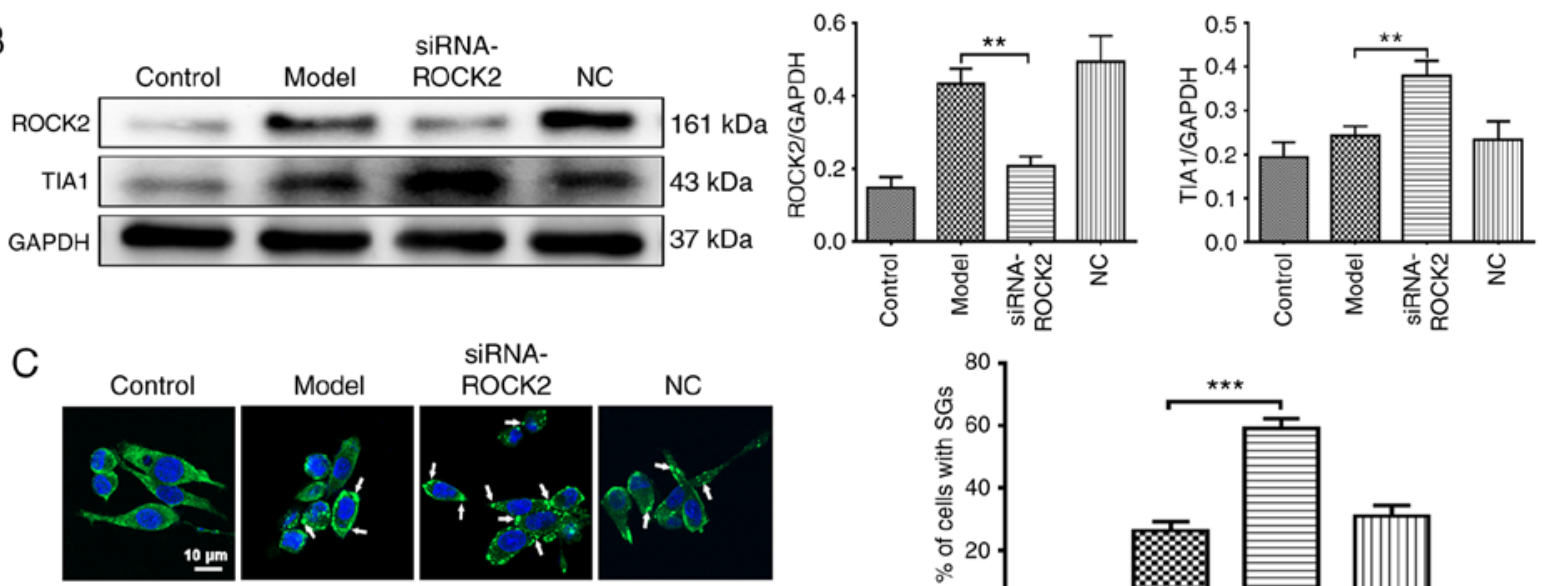

SiRNA-

NC
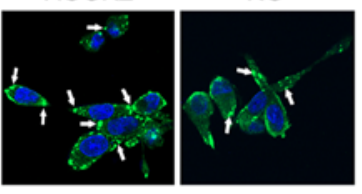

TIA1/
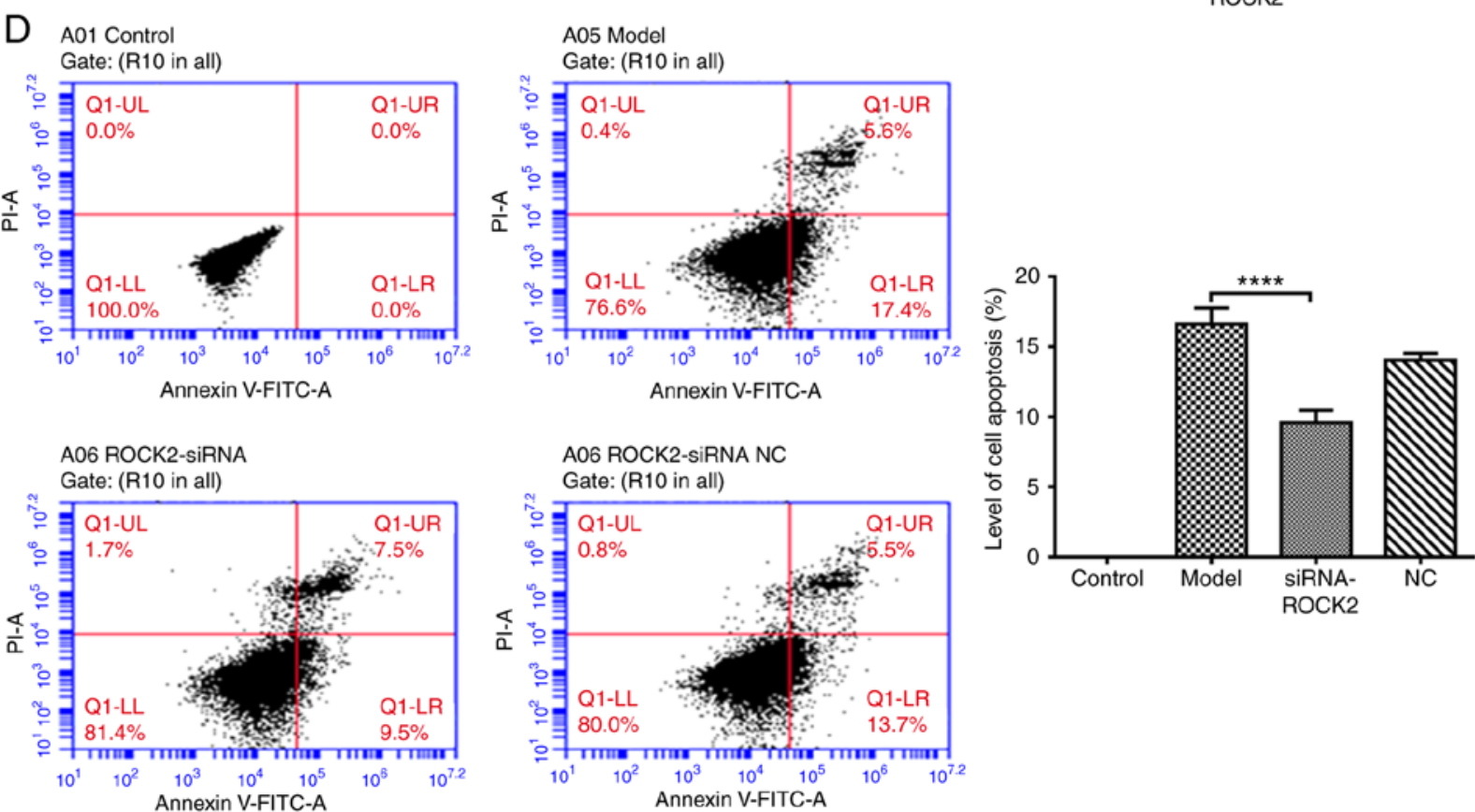

Figure 7. ROCK 2 silencing enhances SG formation and attenuates apoptosis levels in the serum-free cell model. (A) siRNA-ROCK2-1 transfection for $6 \mathrm{~h}$ was the most efficient in reducing ROCK2 expression. PC12 cells were transfected with diverse siRNA-ROCK2 segments at a $100 \mathrm{nM}$ final concentration in 6-well plates. ${ }^{* * *} \mathrm{P}<0.001$ vs. the control group. (B) ROCK2 silencing reduced ROCK2 expression and increased TIA1 expression in the serum-free cell model. Transfection of siRNA-ROCK2-1 and siRNA-ROCK2 NC into cells at a $100 \mathrm{nM}$ final concentration in 6-well plates. After serum-free stimulation for $24 \mathrm{~h}$, PC12 cells were collected for western blotting analysis. ${ }^{* *} \mathrm{P}<0.01$ vs. the model group. (C) ROCK2 silencing promoted SG formation in the serum-free cell model. ${ }^{* * * *} \mathrm{P}<0.001 \mathrm{vs}$. the model group. (D) ROCK2 silencing suppressed cell apoptosis in serum-free cells. Flow cytometry analysis was used for detection. ${ }^{* * * * *} \mathrm{P}<0.0001$ vs. the model group. Data were presented as the mean \pm standard error of the mean. One-way analysis of variance test. Untransfected cells were used as the control group. Each experiment was replicated 3 times. Scale bars, $10 \mu \mathrm{m}$. ROCK2, Rho associated protein kinase 2; SG, stress granules; siRNA, small interfering RNA.

was identified when comparing these two groups $(\mathrm{P}=0.7818$; Fig. 8C). These results indicated that miR-335 specifically targeted the ROCK2 3'-UTR to promote SG formation, reduce ROCK2 expression and suppress cell apoptosis.

\section{Discussion}

Ischemic brain injury is mediated by multiple mechanisms (33) and SG assembly may serve a protective role in the delayed 
A

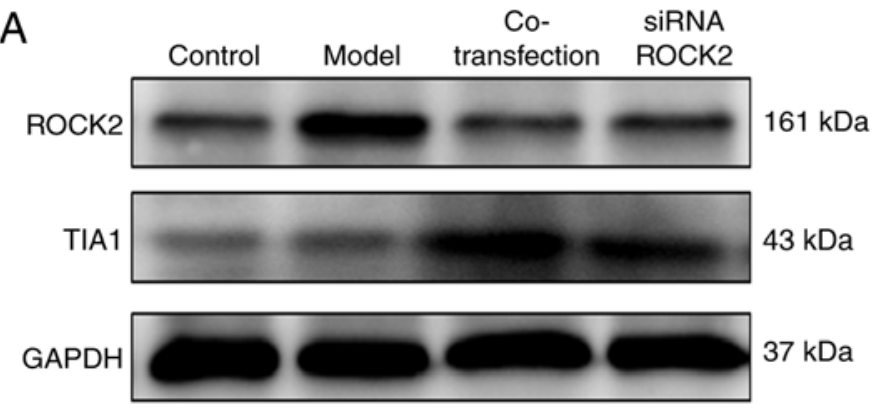

B

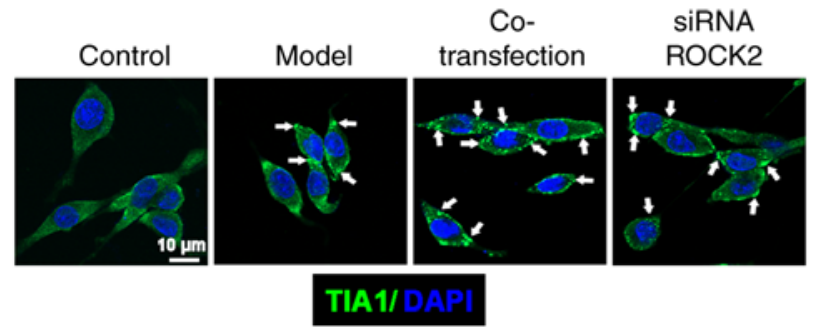

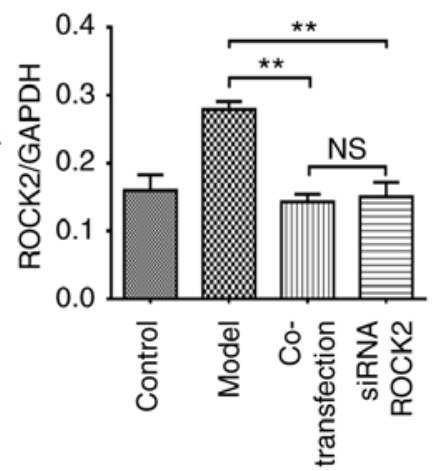
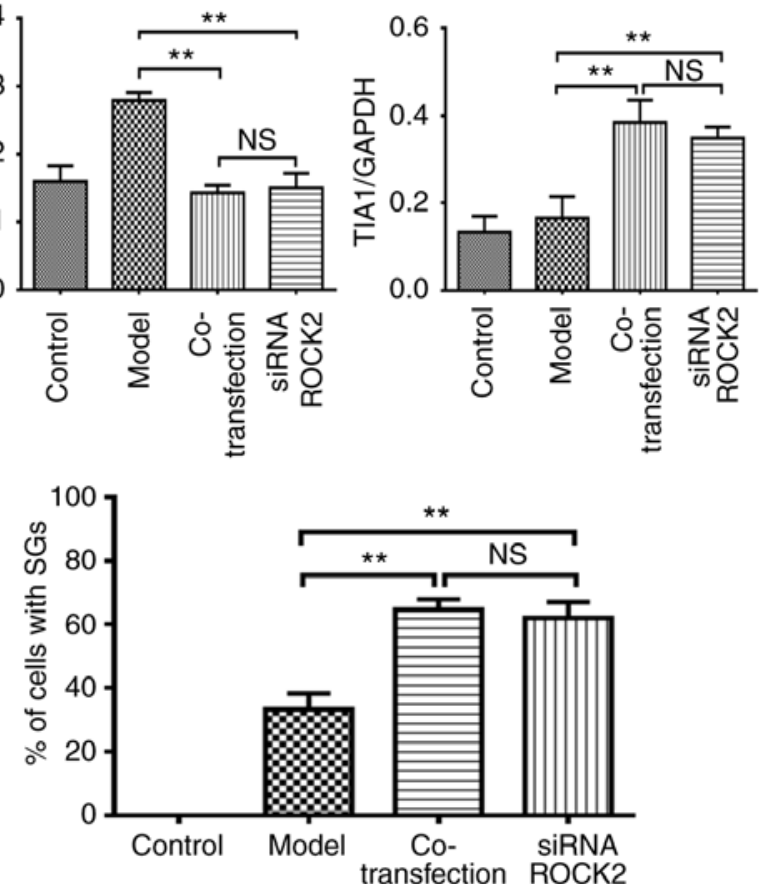

C
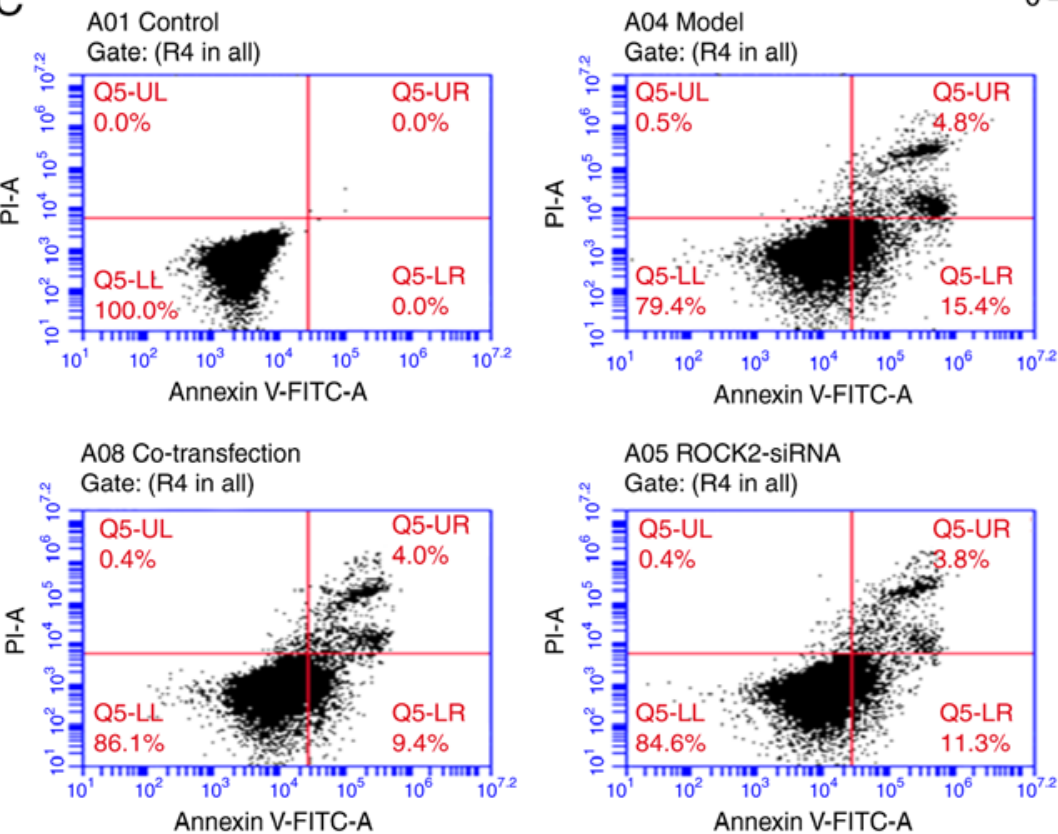

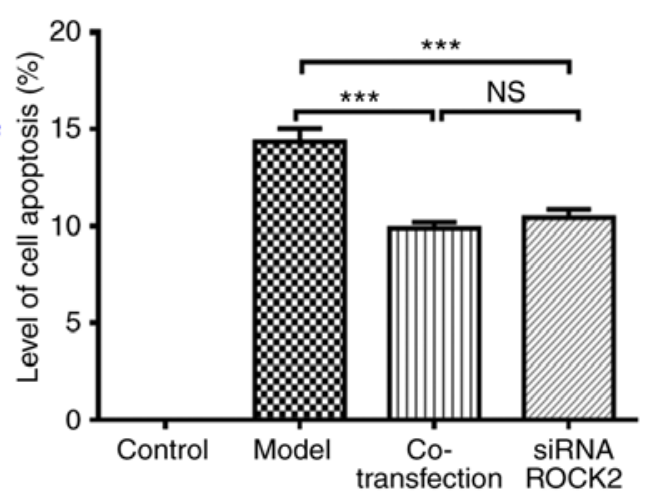

Figure 8. ROCK2 silencing significantly attenuates the effect of miR-335 on SG formation and apoptosis. (A) miR-335 had no significant effect on ROCK2 and TIA1 protein expression following ROCK2 silencing. PC12 cells were transfected with siRNA-ROCK2 (100 nM) or miR-335 mimic (50 nM) for $6 \mathrm{~h}$ in 6 -well plates $\left(1 \times 10^{5}\right.$ cells per well). ${ }^{* *} \mathrm{P}<0.01$ vs. the model group. (B) ROCK2 silencing attenuated the effect of miR-335 on $\mathrm{SG}$ formation. ${ }^{* *} \mathrm{P}<0.01$, vs. the model group. (C) ROCK2 silencing significantly attenuated the effect of miR-335 on apoptosis. Flow cytometry analysis was used for detection. Data were presented as the mean \pm standard error of the mean. ${ }^{* * * *} \mathrm{P}<0.001$ vs. the model group. Each experiment was replicated 3 times. One-way analysis of variance test. Untransfected cells were used as the control group. Scale bars, $10 \mu \mathrm{m}$. ROCK2, Rho associated coiled-coil containing protein kinase 2; TIA1, T-cell intracellular antigen-1; SG, stress granules; miR, microRNA; NS, no significant differences.

progression of ischemic brain injury. However, few studies have focused on the roles of miRNAs in the SG assembly of stroke. The present study used MCAO and serum-free cell models to mimic various pathological features in the early stages of stroke to further investigate the roles of miRNAs in SG assembly in AIS. The major results of the present study were as follows: i) An inverse association between SG formation and apoptotic level was identified in MCAO rats; ii) miR-335 was decreased in the MCAO rats and the mRNA of ROCK2 was the effective target of miR-335; and iii) the SG formation promoted by miR-335 inhibits apoptosis levels by inhibiting ROCK 2 expression in AIS. The results also demonstrated that the miR-335-ROCK2 signaling pathway may serve an important role in SG formation and apoptosis levels in stroke and this pathway may be a pivotal target for treatment in AIS.

An important result of the present study was that an inverse association between SG formation and apoptosis was observed in MCAO rats. Previous studies have demonstrated that TIA1 expression could be used as a hallmark for SG presence (34-36). SG formation peaked at $6 \mathrm{~h}$ following reperfusion and decreased at $24 \mathrm{~h}$ after reperfusion; apoptosis levels were 
the lowest at $6 \mathrm{~h}$ post-reperfusion and peaked at $24 \mathrm{~h}$ following reperfusion. These observations suggest that decreasing SG formation may promote apoptotic levels and aggravate brain injury in MCAO rats. These results are consistent with a recent study that revealed that SGs have a dynamic structure and exert an anti-apoptosis function in stressed cells (37). The results of the present study suggested that the dynamic regulation of SGs may serve as a protective pathway in stroke.

Another important finding of present study was that miR-335 promoted SG formation in MCAO rats and PC12 cells with serum-free stimulation. Microarray data and qPCR detection identified that miR-335 was specifically reduced in MCAO rats. These results are consistent with the results of previous study that suggested that dysregulation of miRNAs can be linked to ischemic stroke (38). Furthermore, it has been reported that miRNAs can affect the formation of SGs (39). However, the role of miR-335 on SG formation in response to stroke remains unknown. The present study demonstrated that miR-335 treatment increased SG formation and reduced the apoptotic level in animal and cell experiments, which protects the brain from ischemic injury. This conclusion was consistent with a previous report (30). Under stress conditions, SG formation enhances cell survival and prevents apoptosis (40). If the stress is persistent, SG will be degraded and followed by apoptosis initiation (41). There have been several studies investigating the association between SG and apoptosis (42-44). Therefore, increasing SG formation promoted by miR-335 may be a therapeutic strategy for stroke. The results of the present study suggested that the target gene silencing function of miR-335 may act as a mediator between SG formation and apoptosis in vivo and in vitro. This finding may indicate the molecular mechanism underlying the anti-apoptotic effect of SG formation in AIS.

In the present study, an important mechanism was revealed as miR-335 was observed to regulate SG formation by targeting the mRNA of ROCK2. Bioinformatics analysis predicted that ROCK 2 mRNA is targeted by miR-335 and ROCK2 served an important role in ischemic stroke $(45,46)$. ROCKs can lead to the phosphorylation of downstream substrates and induce alterations in cellular function. The present study demonstrated that ROCK2 increased along with the elevated levels of apoptosis and was negatively correlated with SG formation in vivo and in vitro. The observations of the present study suggest that ROCK2 functions as a mediator between apoptosis and SG formation. To verify ROCK2 functions in cells, RNAi experiments were performed with ROCK2 silencing and miR-335 co-transfection in this study. The results of the present study confirm that ROCK 2 inhibited the expression of TIA1, which resulted in the reduction of $\mathrm{SG}$ formation and increased apoptosis level. In previous studies (47-50), the association between the dynamic generation of SG and the post-translational modification of crucial RBPs has been investigated. These studies demonstrated that SG nucleating factors can be phosphorylated in the serine and threonine residues by specific kinases, which generally reduce the interactions between RBPs and mRNAs, and finally decrease SG assembly. It should be noted that miR-335 inhibited TIA1 phosphorylation in MCAO rats in the present study. This may be explained by the potential mechanism that the serine/threonine residue sites of TIA1 may be phosphorylated by the kinase activity of ROCK2, which in turn impacts the assembly of SG under stress conditions.

There are several important clinical implications of these results. Firstly, miR-335 may be a potential biomarker for the early diagnosis of stroke. miR-335 was decreased in the ischemic cortices of MCAO rats, which is consistent with a previous study which revealed that plasma miR-335 expression decreased in patients with AIS (51). Secondly, there are currently few therapies to protect against the progression of stroke. The present study has revealed that miR-335 inhibited apoptosis by promoting SG formation in AIS. Finally, it is important to identify therapeutic targets for the effective protection against cerebral ischemic impairments. In conclusion, the results of the present study support novel potential therapeutic targets for AIS based on the regulation of the miR-335-ROCK2 signaling pathway in SG formation.

\section{Acknowledgements}

Not applicable.

\section{Funding}

The present study was supported by the National Natural Science Foundation of China (grant no. 81673770) and the National Natural Science Foundation of Guangdong province (grant no. 2017A030312009).

\section{Availability of data and materials}

The data and materials generated during the study are available from the corresponding author on reasonable request.

\section{Authors' contributions}

WS performed the experiments and wrote the manuscript. SY performed the experiments and analyzed the data. ZR, XL, ZW and YL performed the cell and animal experiments. $\mathrm{JZ}$ and RD provided the reagents/materials. SZ performed the histological examinations of the brain. YwL designed the experiments and analyzed the data. DC conceived and designed the experiments. All authors have read and approved the final manuscript.

\section{Ethics approval and consent to participate}

All animal experiments were approved by the Care and Use of Experimental Animals Committee of Guangzhou University of Chinese Medicine and performed according to the National Institute of Health Guide for the Care and Use of Laboratory Animals.

\section{Patient consent for publication}

Not applicable.

\section{Competing interest}

All authors declare that they have no competing interest to disclose. 


\section{References}

1. Gutierrez-Beltran E, Moschou PN, Smertenko AP and Bozhkov PV: Tudor staphylococcal nuclease links formation of stress granules and processing bodies with mRNA catabolism in arabidopsis. Plant Cell 27: 926-943, 2015.

2. Takahashi M, Higuchi M, Matsuki H, Yoshita M, Ohsawa T, Oie $\mathrm{M}$ and Fujii M: Stress granules inhibit apoptosis by reducing reactive oxygen species production. Mol Cell Biol 33: 815-829, 2013.

3. Arimoto-Matsuzaki K, Saito H and Takekawa M: TIA1 oxidation inhibits stress granule assembly and sensitizes cells to stress-induced apoptosis. Nat Commun 7: 10252, 2016.

4. Waris S, Wilce MC and Wilce JA: RNA recognition and stress granule formation by TIA proteins. Int J Mol Sci 15: 23377-23388, 2014.

5. Sampuda KM, Riley M and Boyd L: Stress induced nuclear granules form in response to accumulation of misfolded proteins in caenorhabditis elegans. BMC Cell Biol 18: 18, 2017.

6. Moretti A, Ferrari F and Villa RF: Pharmacological therapy of acute ischaemic stroke: Achievements and problems. Pharmacol Ther 153: 79-89, 2015.

7. Wei G, Chen YB, Chen DF, Lai XP, Liu DH, Deng RD, Zhou JH, Zhang SX, Li YW, Lii H, et al: $\beta$-Asarone inhibits neuronal apoptosis via the CaMKII/CREB/Bcl-2 signaling pathway in an in vitro model and A $\beta P P / P S 1$ mice. J Alzheimers Dis 33: 863-880, 2013.

8. Radak D, Katsiki N, Resanovic I, Jovanovic A, Sudar-Milovanovic E, Zafirovic S, Mousad SA and Isenovic ER Apoptosis and acute brain ischemia in ischemic stroke. Curr Vasc Pharmacol 15: 115-122, 2017.

9. Zhu H, Gui Q, Hui X, Wang X, Jiang J, Ding L, Sun X, Wang Y and Chen $\mathrm{H}$ : TGF- $\beta 1 /$ smad3 signaling pathway suppresses cell apoptosis in cerebral ischemic stroke rats. Med Sci Monit 23: 366-376, 2017.

10. Li SH, Chen L, Pang XM, Su SY, Zhou X, Chen CY, Huang LG, Li JP and Liu JL: Decreased miR-146a expression in acute ischemic stroke directly targets the Fbx110 mRNA and is involved in modulating apoptosis. Neurochem Int 107: 156-167, 2017.

11. Liao JK, Seto M and Noma K: Rho kinase (ROCK) inhibitors. J Cardiovasc Pharmacol 50: 17-24, 2007.

12. Riento K and Ridley AJ: Rocks: Multifunctional kinases in cell behaviour. Nat Rev Mol Cell Biol 4: 446-456, 2003.

13. Sebbagh M, Hamelin J, Bertoglio J, Solary E and Breard J: Direct cleavage of ROCK II by granzyme B induces target cell membrane blebbing in a caspase-independent manner. J Exp Med 201: 465-471, 2005

14. Lowery DM, Clauser KR, Hjerrild M, Lim D, Alexander J, Kishi K, Ong SE, Gammeltoft S, Carr SA and Yaffe MB Proteomic screen defines the Polo-box domain interactome and identifies Rock2 as a Plk1 substrate. EMBO J 26: 2262-2273, 2007.

15. Ma Z, Kanai M,Kawamura K, Kaibuchi K, Ye K and Fukasawa K Interaction between ROCK II and nucleophosmin/B23 in the regulation of centrosome duplication. Mol Cell Biol 26: 9016-9034, 2006.

16. Meekins LC, Rosado-Adames N, Maddala R, Zhao JJ, Rao PV and Afshari NA: Corneal endothelial cell migration and proliferation enhanced by rho kinase (ROCK) inhibitors in in vitro and in vivo models. Invest Ophthalmol Vis Sci 57: 6731-6738, 2016.

17. Pan P, Shen M, Yu H, Li Y, Li D and Hou T: Advances in the development of Rho-associated protein kinase (ROCK) inhibitors. Drug Discov Today 18: 1323-1333, 2013.

18. LoGrasso PV and Feng Y: Rho kinase (ROCK) inhibitors and their application to inflammatory disorders. Curr Top Med Chem 9: 704-723, 2009

19. Li F, Wei G, Bai Y, Li Y, Huang F, Lin J, Hou Q, Deng R, Zhou JH, Zhang SX and Chen DF: MicroRNA-574 is involved in cognitive impairment in 5-month-old APP/PS1 mice through regulation of neuritin. Brain Res 1627: 177-188, 2015.

20. Jickling GC, Ander BP, Zhan X, Noblett D, Stamova B and Liu D: microRNA expression in peripheral blood cells following acute ischemic stroke and their predicted gene targets. PLoS One 9: e99283, 2014.

21. Jolana L and Kamil D: The role of microRNA in ischemic and hemorrhagic stroke. Curr Drug Deliv 14: 816-831, 2017.

22. Koutsis G, Siasos G and Spengos K: The emerging role of microRNA in stroke. Curr Top Med Chem 13: 1573-1588, 2013.
23. Chen QY, Liu N, Ma J, Fang Y, Cao Y, Li H and Liu YC: Effect of a pre-microRNA-149 (miR-149) genetic variation on the risk of ischemic stroke in a chinese han population. Genet Mol Res 14: 2582-2589, 2015

24. Wei N, Xiao L, Xue R, Zhang D, Zhou J, Ren H, Guo S and Xu J: MicroRNA-9 mediates the cell apoptosis by targeting Bcl2111 in ischemic stroke. Mol Neurobiol 53: 6809-6817, 2016.

25. Liu W, Chen X and Zhang Y: Effects of microRNA-21 and microRNA-24 inhibitors on neuronal apoptosis in ischemic stroke. Am J Transl Res 8: 3179-3187, 2016.

26. Sun Y, Gui H, Li Q, Luo ZM, Zheng MJ, Duan JL and Liu X: MicroRNA-124 protects neurons against apoptosis in cerebral ischemic stroke. CNS Neurosci Ther 19: 813-819, 2013.

27. Liu X, Li F, Zhao S, Luo Y, Kang J, Zhao H, Yan F, Li S and Ji X: MicroRNA-124-mediated regulation of inhibitory member of apoptosis-stimulating protein of p53 family in experimental stroke. Stroke 44: 1973-1980, 2013.

28. Bleilevens C, Roehl AB, Goetzenich A, Zoremba N, Kipp M, Dang J, Tolba R, Rossaint R and Hein M: Effect of anesthesia and cerebral blood flow on neuronal injury in a rat middle cerebral artery occlusion (MCAO) model. Exp Brain Res 224: 155-164, 2013.

29. Yang X, Liu Y, Liu C, Xie W, Huang E, Huang W, Wang J, Chen L, Wang H, Qiu P, et al: Inhibition of ROCK2 expression protects against methamphetamine-induced neurotoxicity in PC12 cells. Brain Res 1533: 16-25, 2013.

30. Liu FJ, Kaur P, Karolina DS, Sepramaniam S, Armugam A, Wong PT and Jeyaseelan K: MiR-335 regulates Hif-1 $\alpha$ to reduce cell death in both mouse cell line and rat ischemic models. PLoS One 10: e0128432, 2015.

31. Xu J and Wong C: A computational screen for mouse signaling pathways targeted by microRNA clusters. RNA 14: 1276-1283, 2008.

32. Wang J, Muheremu A, Zhang M, Gong K, Huang C, Ji Y, Wei Y and Ao Q: MicroRNA-338 and microRNA-21 co-transfection for the treatment of rat sciatic nerve injury. Neurol Sci 37: 883-890, 2016.

33. Chi W, Meng F, Li Y, Li P, Wang G, Cheng H, Han S and Li J: Impact of microRNA-134 on neural cell survival against ischemic injury in primary cultured neuronal cells and mouse brain with ischemic stroke by targeting HSPA12B. Brain Res 1592: 22-33, 2014

34. Bley N, Lederer M, Pfalz B, Reinke C, Fuchs T, Glaß M, Möller B and Hüttelmaier S: Stress granules are dispensable for mRNA stabilization during cellular stress. Nucleic Acids Res 43: e26, 2015.

35. Gilks N, Kedersha N, Ayodele M, Shen L, Stoecklin G, Dember LM and Anderson P: Stress granule assembly is mediated by prion-like aggregation of TIA-1. Mol Biol Cell 15: 5383-5398, 2004

36. Wolczyk M, Podszywalow-Bartnicka P, Bugajski L and Piwocka K: Stress granules assembly affects detection of mRNA in living cells by the NanoFlares; An important aspect of the technology. Biochim Biophys Acta Gen Subj 1861: 1024-1035, 2017.

37. Wheeler JR, Matheny T, Jain S, Abrisch R and Parker R: Distinct stages in stress granule assembly and disassembly. ELife 5: e18413, 2016.

38. Dharap A, Bowen K, Place R, Li LC and Vemuganti R: Transient focal ischemia induces extensive temporal changes in rat cerebral microRNAome. J Cereb Blood Flow Metab 29: 675-687, 2009.

39. Pothof J, Verkaik NS, Hoeijmakers JH and van Gent DC: MicroRNA responses and stress granule formation modulate the DNA damage response. Cell Cycle 8: 3462-3468, 2009.

40. Tsai NP and Wei LN: RhoA/ROCK1 signaling regulates stress granule formation and apoptosis. Cell Signal 22: 668-675, 2010.

41. Qi D, Huang S, Miao R, She ZG, Quinn T, Chang Y, Liu J, Fan D, Chen YE and Fu M: Monocyte chemotactic protein-induced protein 1 (MCPIP1) suppresses stress granule formation and determines apoptosis under stress. J Biol Chem 286: 41692-41700, 2011.

42. Nikpour P, Baygi ME, Steinhoff C, Hader C, Luca AC, Mowla SJ and Schulz WA: The RNA binding protein musashil regulates apoptosis, gene expression and stress granule formation in urothelial carcinoma cells. J Cell Mol Med 15: 1210-1224, 2011.

43. Eisinger-Mathason TS, Andrade J, Groehler AL, Clark DE, Muratore-Schroeder TL, Pasic L, Smith JA, Shabanowitz J, Hunt DF, Macara IG and Lannigan DA: Codependent functions of RSK2 and the apoptosis-promoting factor TIA-1 in stress granule assembly and cell survival. Mol Cell 31: 722-736, 2008. 
44. Gareau C, Fournier MJ, Filion C, Coudert L, Martel D, Labelle Y and Mazroui R: p21(WAF1/CIP1) upregulation through the stress granule-associated protein CUGBP1 confers resistance to bortezomib-mediated apoptosis. PLoS One 6: e20254, 2011.

45. Feske SK, Sorond FA, Henderson GV, Seto M, Hitomi A, Kawasaki K, Sasaki Y, Asano T and Liao JK: Increased leukocyte ROCK activity in patients after acute ischemic stroke. Brain Res 1257: 89-93, 2009.

46. Sladojevic N, Yu B and Liao JK: ROCK as a therapeutic target for ischemic stroke. Expert Rev Neurother 17: 1167-1177, 2017.

47. Su C, Gao X, Yang W, Zhao Y, Fu X, Cui X, Zhang C, Xin L, Ren Y, Li L, et al: Phosphorylation of Tudor-SN, a novel substrate of JNK, is involved in the efficient recruitment of Tudor-SN into stress granules. Biochim Biophys Acta Mol Cell Res 1864: 562-571, 2017.

48. Yoon JH, Abdelmohsen K, Srikantan S, Guo R, Yang X, Martindale JL and Gorospe M: Tyrosine phosphorylation of HuR by JAK3 triggers dissociation and degradation of HuR target mRNAs. Nucleic Acids Res 42: 1196-1208, 2014.
49. Reineke LC, Tsai WC, Jain A, Kaelber JT, Jung SY and Lloyd RE: Casein kinase 2 is linked to stress granule dynamics through phosphorylation of the stress granule nucleating protein G3BP1. Mol Cell biol 37: e00596-e00616, 2017.

50. Eberhardt W, Doller A and Pfeilschifter J: Regulation of the mRNA-binding protein HuR by posttranslational modification: Spotlight on phosphorylation. Curr Protein Pept Sci 13: 380-390, 2012.

51. Zhao B,Zhu Z,Hao J, Wan Z and Guo X: Decreased plasma miR-335 expression in patients with acute ischemic stroke and its association with calmodulin expression. J Int Med Res 44: 1331-1338, 2016.

This work is licensed under a Creative Commons Attribution-NonCommercial-NoDerivatives 4.0 International (CC BY-NC-ND 4.0) License. 